\title{
Thermal instability revisited
}

\author{
S. A. E. G. Falle ${ }^{1}{ }^{\star}$ C. J. Wareing ${ }^{2}$ and J. M. Pittard ${ }^{2}$ \\ ${ }^{1}$ Department of Applied Mathematics, University of Leeds, Leeds LS2 9JT, UK \\ ${ }^{2}$ School of Physics and Astronomy, University of Leeds, Leeds LS2 9JT, UK
}

Accepted XXX. Received YYY; in original form ZZZ

\begin{abstract}
Field's linear analysis of thermal instability is repeated using methods related to Whitham's theory of wave hierarchies, which brings out the physically relevant parameters in a much clearer way than in the original analysis. It is also used for the stability of non-equilibrium states and we show that for gas cooling behind a shock, the usual analysis is only quantitatively valid for shocks that are just able to trigger a transition to the cold phase. A magnetic field can readily be included and we show that this does not change the stability criteria. By considering steady shock solutions, we show that almost all plausible initial conditions lead to a magnetically dominated state on the unstable part of the equilibrium curve. These results are used to analyse numerical calculations of perturbed steady shock solutions and of shocks interacting with a warm cloud.
\end{abstract}

Key words: Hydrodynamics - MHD - instabilities - shock waves - methods: analytic

\section{INTRODUCTION}

It is very common for astrophysical plasmas to be subject to heating and cooling processes. If these are sufficiently rapid compared to other relevant timescales, then the plasma will be in thermal equilibrium and if this is unstable, then we have a possible mechanism for generating density inhomogeneities that does not rely on self-gravity. This motivated Field (1965) to consider the linear stability of such equilibrium states and to apply his results to the solar chromosphere and corona, planetary nebulae, the galactic halo and galaxy formation. As discussed in Field et al. (1969) and McKee \& Ostriker (1977), thermal instability is also a key ingredient in multiphase models of the ISM. Since then there have been numerous papers that have considered the effect of thermal instability in a diverse range of situations, such as solar prominences, e.g. Xia \& Keppens (2016), star forming regions, e.g. Kim et al. (2008), broad-line regions in active galaxies, e.g. Begelman \& McKee (1990) and the circumgalactic medium, e.g. Stern et al. (2016). The analysis has also been extended to include perturbations of nonequilibrium states (e.g. Schwarz et al. 1972; Balbus 1986; Koyama \& Inutsuka 2000).

Recently, Waters \& Proga (2019) have revisited Field's analysis and written the dispersion relation in a somewhat different form. Their paper includes a discussion of the various modes of instability, together with numerical calculations of the non-linear evolution of the condensation mode. They also consider non-equilibrium initial states.
In view of the above, one might suppose that there is little point in revisiting the linear analysis of either the equilibrium or non-equilibrium states. However, there is a more modern method of analysing such a linear dispersion relation based on ideas from control theory and the Whitham theory of wave hierarchies (Whitham 1974). Although this yields few new results, it does illuminate the physics rather more clearly than the traditional approach.

The analysis is described in Section 2 and it is applied to the energy source function proposed by Koyama \& Inutsuka (2002) in Section 3. In Section 4 these results are used to analyse numerical calculations of shock interactions and the work is summarised in Section 5.

\section{HYPERBOLIC BALANCE LAWS}

A system of hyperbolic balance laws in one space dimension is of the form

$\partial_{t} \mathbf{u}+\partial_{x} \mathbf{f}(\mathbf{u})=\mathbf{s}(\mathbf{u})$,

where $\mathbf{u}=\left(u_{1}, \cdots u_{n}\right)^{t}$ are a set of $n$ conserved quantities, $\mathbf{f}(\mathbf{u})=\left(f_{1}, \cdots f_{n}\right)^{t}$ are the associated fluxes and $\mathbf{s}(\mathbf{u})=$ $\left(s_{1}, \cdots s_{n}\right)^{t}$ are source terms depending upon $\mathbf{u}$. Here the superfix $t$ denotes the transpose.

For sufficently short wavelengths, the derivatives dominate over the source terms and we have a frozen system in which $\mathbf{s}$ can be neglected. For long wavelengths the source term dominates and we have

$\mathbf{s}=0$. 
This imposes $r$ conditions on $\mathbf{u}$ where $r$ is the rank of $\mathbf{s}$. The system is then reduced to an equilibrium system with $n-r$ variables described by $\mathbf{u}_{e}=\mathbf{u}_{e}(\mathbf{u})$. The Whitham theory only considers the case $r=1$, but it is possible to extend it to $r>1$ (Falle \& Williams 2019).

\subsection{Gas dynamics with an energy source}

For gas dynamics with an energy source term, we have

$\mathbf{u}=[\rho, \rho v, e]^{t}, \quad \mathbf{f}=\left[\rho v, p+\rho v^{2}, v(e+p)\right]^{t}, \quad \mathbf{s}=[0,0,-\rho L]^{t}$,

where $\rho, v, p$ are the density, velocity and pressure.

$e=\frac{p}{(\gamma-1)}+\frac{1}{2} \rho v^{2}$

is the total energy per unit volume and $L(\rho, T)$ is the energy loss rate per unit mass. Clearly $r=1$ in this case.

It is more convenient to write these in the form

$\partial_{t} \mathbf{p}+\mathrm{A} \partial_{x} \mathbf{p}=(\gamma-1) \mathbf{s}$,

where

$\mathbf{p}=[\rho, v, p]^{t}$,

are the primitive variables and

$\mathrm{A}=\left(\begin{array}{ccc}v & \rho & 0 \\ 0 & v & 1 / \rho \\ 0 & \gamma p & v\end{array}\right)$.

We now assume a solution of the form

$\mathbf{p}=\mathbf{p}_{0}+\mathbf{p}_{1} \exp (i \omega t-i k x)$,

where $\mathbf{p}_{0}$ is an equilibrium state with $v=0, L\left(\rho_{0}, T_{0}\right)=0$ and $\mathbf{p}_{1}$ is a small perturbation. The linearised equations then give

$i \omega \mathbf{p}_{1}-i k \mathrm{~A}_{0} \mathbf{p}_{1}=\mathrm{D}_{0} \mathbf{p}_{1}$,

where $A_{0}=A\left(\rho_{0}, T_{0}\right)$ and

$\mathrm{D}_{0}=\left(\begin{array}{ccc}0 & 0 & 0 \\ 0 & 0 & 0 \\ -\rho_{0} G_{\rho}+p_{0} G_{T} / \rho_{0} & 0 & -G_{T}\end{array}\right)$,

with

$$
\begin{aligned}
& G_{\rho}=(\gamma-1) L_{\rho}=(\gamma-1)\left(\frac{\partial L}{\partial \rho}\right)_{T}, \\
& G_{T}=(\gamma-1) L_{T}=(\gamma-1) \frac{m}{k_{B}}\left(\frac{\partial L}{\partial T}\right)_{\rho},
\end{aligned}
$$

where $k_{B}$ is Boltzmann's constant and $m$ is the mean particle mass. The eigenvalues of D are 0,0 and $-G_{T}$, so that we have isochoric instability if

$G_{T}<0 \Rightarrow L_{T}<0$,

which is equation (4a) in Field (1965). From now on we will assume that $G_{T}>0$.

The dispersion relation associated with (9) is

$\left|\omega \mathrm{I}-k \mathrm{~A}_{0}+i \mathrm{D}_{0}\right|=0$,

where $\mathrm{I}$ is the identity matrix. This can be written

$P=P_{0}-\frac{i}{k} P_{1}=0$, where

$P_{0}=\mu\left(\mu^{2}-a_{f}^{2}\right), \quad P_{1}=G_{T}\left(\mu^{2}-a_{e}^{2}\right), \quad \mu=\frac{\omega}{k}$.

Here the frozen sound speed, $a_{f}$, applies in the adiabatic case and the equilibrium sound speed, $a_{e}$, when the system is in thermal equilibrium i.e. equation (2) is satisfied. These are given by

$a_{f}^{2}=\frac{\gamma p_{0}}{\rho_{0}}, \quad a_{e}^{2}=\left(\frac{\partial p_{0}}{\partial \rho_{0}}\right)_{L=0}=\frac{G_{T} a_{f}^{2}-\rho_{0} \gamma G_{\rho}}{\gamma G_{T}}$.

$P_{0}$ describes the frozen system since its roots are the wave speeds of the frozen system, $0, \pm a_{f}$, whereas $P_{1}$ describes the equilibrium system since its roots, $\pm a_{e}$, are the wave speeds of the equilibrium system. If we define the acoustic or thermal wavenumber by

$k_{T}=\frac{G_{T}}{a_{f}}=\frac{(\gamma-1) m L_{T}}{k_{B} a_{f}}$,

then the source term is neglible for $k \gg k_{T}$ and we have the frozen system whereas the source term dominates and enforces equilibrium for $k \ll k_{T}$. This is the same as $k_{T}$ in equation (16) in Field (1965). The corresponding wavelength is

$\lambda_{T}=\frac{2 \pi}{k_{T}}=\frac{2 \pi k_{B} a_{f}}{(\gamma-1) m L_{T}}$

This separation of the dispersion relation into polynomials corresponding to the frozen and equilibrium systems was first used by Whitham (1974) in his theory of wave hierarchies. As he shows, it can be applied to many different systems, but to our knowledge has only been used in an astrophysical context by Tytarenko et al. (2002).

The Hermite-Biehler theorem (e.g. Bhattacharyya et al. 1995; Tytarenko et al. 2002; Falle \& Williams 2019) tells us that if the coefficients of the highest power of $\mu$ in $P_{0}$ and $P_{1}$ have the same sign, the roots of $P_{0}$ and $P_{1}$ are real and the roots of $P_{1}$ interleave with those of $P_{0}$, then the roots of (14) all have positive imaginary parts and the system is stable. This stability condition is both necessary and sufficient.

The system will certainly be unstable if $a_{e}^{2}$ is imaginary, which requires

$a_{e}^{2}=\frac{G_{T} a_{f}^{2}-\rho_{0} \gamma G_{\rho}}{\gamma G_{T}}<0 \Rightarrow 1-\frac{\rho_{0}}{T_{0}} \frac{L_{\rho}}{L_{T}}<0$,

from equation (16). This is the isobaric instability condition, equation (4b) in Field (1965) when $L_{T}>0$.

For real $a_{e}$, the roots of $P_{0}$ and $P_{1}$ do not interleave if

$a_{e}>a_{f} \Rightarrow a_{f}^{2}-a_{e}^{2}=(\gamma-1) \frac{p_{0}}{\rho_{0}}+\frac{\rho_{0} G_{\rho}}{G_{T}}=(\gamma-1) \frac{p_{0}}{\rho_{0}}+\frac{k_{B} \rho_{0} L_{\rho}}{m L_{T}}<0$

This is the isentropic instability condition for sound waves, equation (5) in Field (1965) when $L_{T}>0$.

In the absence of conduction, the only dimensionless parameter is

$\alpha=\frac{a_{e}^{2}}{a_{f}^{2}}$.

This plays the same role as the dimensionless parameter, $\alpha$, 
in Field (1965), but has a more obvious physical significance. In particular, the stability conditions take the simple form

$$
\begin{array}{ll}
\alpha<0 & \text { isobaric instability, } \\
0 \leq \alpha \leq 1 & \text { stable, } \\
\alpha>1 & \text { isentropic instability. }
\end{array}
$$

Now consider the behaviour of the root, $\mu=-a_{e}$, for small $k$. We get

$$
\omega=-a_{e} k+i k^{2} \frac{\left(a_{f}^{2}-a_{e}^{2}\right)}{2 G_{T}}+k^{3} \frac{5 a_{e}^{4}-6 a_{e}^{2} a_{f}^{2}+a_{f}^{4}}{8 G_{T}^{2} a_{f}}+O\left(k^{4}\right),
$$

which corresponds to instability when $a_{e}^{2}<0$.

Similarly, for large $k$, the root $\mu=0$, which corresponds to the non-propagating condensation mode, is given by

$\omega=\frac{i a_{e}^{2} G_{T}}{a_{f}^{2}}+\frac{i a_{e}^{4} G_{T}^{3}\left(a_{f}^{2}-a_{e}^{2}\right)}{k^{2} a_{f}^{8}}+O\left(1 / k^{4}\right)$.

Again we have instability if $a_{e}^{2}<0$. The first term agrees with equation (31) in Field (1965). This tells us that the growth rate tends to a constant as $k \rightarrow \infty$ and that the largest wavenumber modes are the most unstable.

\subsection{Thermal conduction}

If thermal conduction is important, then the pressure equation in (5) becomes

$\partial_{t} p+\gamma p \partial_{x} v+v \partial_{x} p=(\gamma-1) \partial_{x} \kappa \partial_{x} T$

where $\kappa$ is the thermal conductivity. (13) then becomes

$\left|\omega \mathrm{I}-k \mathrm{~A}_{0}+i \mathrm{D}_{0}-i k^{2} \mathrm{C}_{0}\right|=0$,

where

$\mathrm{C}_{0}=\left(\begin{array}{ccc}0 & 0 & 0 \\ 0 & 0 & 0 \\ -\kappa^{\prime} p_{0} / \rho_{0}^{2} & 0 & \kappa^{\prime} / \rho_{0}\end{array}\right)$,

with

$\kappa^{\prime}=(\gamma-1) \frac{m}{k_{B}} \kappa$.

Equation (14) becomes

$P=P_{0}-\frac{i}{k} P_{1}-i k P_{2}=0$,

where

$P_{2}=\frac{\kappa^{\prime}}{\rho_{0}}\left(\mu^{2}-a_{f}^{2} / \gamma\right)=\frac{\kappa^{\prime}}{\rho_{0}}\left(\mu^{2}-c_{T}^{2}\right)$,

and

$c_{T}=\left(\frac{p_{0}}{\rho_{0}}\right)^{1 / 2}$

is the isothermal sound speed. One would expect $P_{2}$ to have this form since it describes the behaviour when $k$ is large enough for thermal conduction to ensure a uniform temperature. We now have three polynomials each associated with a different physical process: $P_{0}$ for the adiabatic system, $P_{1}$ when the energy source term dominates and $P_{2}$ when thermal conduction dominates. Liubarskii (1961) calls these the auxiliary polynomials.

For $\gamma>1$, the roots of $P_{2}$ interleave with those of $P_{0}$ and thermal conduction is stabilising. If the system is subject to the isobaric instability, then $a_{e}^{2}<0$ and conduction stabilises wavenumbers for which

$\frac{G_{T} a_{e}^{2}}{k}+\frac{k \kappa^{\prime} a_{f}^{2}}{\rho_{0} \gamma}>0$

i.e.

$k>k_{F}=\left(\frac{-a_{e}^{2} \gamma \rho_{0} G_{T}}{a_{f}^{2} \kappa^{\prime}}\right)^{1 / 2}=\left(\frac{-\alpha \gamma \rho_{0} L_{T}}{\kappa}\right)^{1 / 2}$.

The corresponding Field length is then

$\lambda_{F}=\frac{2 \pi}{k_{F}}=2 \pi\left(\frac{\kappa}{-\alpha \gamma \rho_{0} L_{T}}\right)^{1 / 2}$,

which agrees with equation (26) in Field (1965) and the expressions in Begelman \& McKee (1990) and Kim et al. (2008). On the other hand, Koyama \& Inutsuka (2004) define

$\lambda_{F}=\left(\frac{T_{0} \kappa}{\rho_{0} L_{c}}\right)^{1 / 2}$,

where $L_{c}$ is the magnitude of the cooling term in $L$. The advantage of (33) is that it really is the linear stability limit. (33) and (34) are in fact very different since the $\lambda_{F}$ defined by (33) goes to infinity at the boundaries of the unstable region, $\left(a_{e}=0\right)$, as it should.

The effect of conduction is determined by the dimensionless parameter

$\beta=\frac{G_{T} \kappa^{\prime}}{\rho a_{f}^{2}}=\frac{(\gamma-1)^{2} m^{2} L_{T} \kappa}{k_{B}^{2} \rho_{0} a_{f}^{2}}$.

The Field wavenumber is then given by

$k_{F}=k_{T}\left(\frac{-\gamma \alpha}{\beta}\right)^{1 / 2}$

Again, this seems to be a more natural choice than the corresponding dimensionless parameter, $\beta$, in Field (1965).

If we define the dimensionless variables

$\bar{\mu}=\frac{\mu}{a_{f}}, \quad \bar{k}=\frac{k a_{f}}{G_{T}}=\frac{k}{k_{T}}$,

then (29) becomes

$\bar{\mu}\left(\bar{\mu}^{2}-1\right)-\frac{i}{\bar{k}}\left(\bar{\mu}^{2}-\alpha\right)-i \beta \bar{k}\left(\bar{\mu}^{2}-1 / \gamma\right)=0$.

If we put

$\bar{\mu}=-i y$,

then (38) becomes

$y\left(y^{2}+1\right)+\frac{1}{\bar{k}}\left(y^{2}+\alpha\right)+\beta \bar{k}\left(y^{2}+1 / \gamma\right)=0$,

which is our version of equation (18) in Field (1965). Note that equation (36) tells us that this has a zero root when $\bar{k}=k_{F} / k_{T}$, as expected.

\subsection{Magnetic field}

The analysis can readily be extended to include an oblique magnetic field with components $B_{x}, B_{y}$. We have the fast and slow magnetosonic speeds,

$c_{f, s}^{2}=\frac{1}{2}\left[a^{2}+B^{2} / \rho \pm \sqrt{ }\left\{\left(a^{2}+B^{2} / \rho\right)^{2}-4 B_{x} a^{2} / \rho\right\}\right]$, 
where $a=a_{f}$ for the frozen system and $a=a_{e}$ for the equilibrium system.

In the absence of conduction, the dispersion relation must now be

$\mu\left(\mu^{2}-c_{f f}^{2}\right)\left(\mu^{2}-c_{f s}^{2}\right)-\frac{i}{k} G_{T}\left(\mu^{2}-c_{e f}^{2}\right)\left(\mu^{2}-c_{e s}^{2}\right)=0$,

where $c_{f f}, c_{f s}$ are the frozen fast/slow speeds and $c_{e f}, c_{e s}$ the equilibrium ones. Since the equilibrium slow speed is imaginary when $a_{e}$ is imaginary, the isobaric instability is still given by (22). Furthermore, the interleaving also fails when $a_{e}>a_{f}$, so that isentropic instability is also governed by (22) i.e. the stability conditions are unchanged. More surprisingly, the growth rate for large $k$ is now

$\omega=\frac{i G_{T} c_{e f}^{2} c_{e s}^{2}}{c_{f f}^{2} c_{f s}^{2}}+O\left(1 / k^{2}\right)=\frac{i a_{e}^{2} G_{T}}{a_{f}^{2}}+O\left(1 / k^{2}\right)$

i.e. exactly the same as (24) for the non-magnetic case. It agrees with the result in Dudorov et al. (2019). Note that for a purely transverse field the slow speed is zero and it is possible for the magnetic field to stabilise the isobaric mode. However, this is a singular case that has a vanishingly small probability of occurring in reality.

This is another illustration of the power of the method: the physics tells how to write down the adiabatic and equilibrium polynomials from what we already know about the wave speeds of the frozen and equilibrium MHD systems. As we have already pointed out, we could also have done this for thermal conduction. The only difficulty is in obtaining the coefficient multiplying the polynomials, but these can often be obtained by inspection of the relevant matrix. This is certainly true for the energy source, thermal conduction and magnetic field.

\subsection{Stability of non-equilibrium states}

Field (1965), Schwarz et al. (1972), Balbus (1986) and Koyama \& Inutsuka (2000) extend the thermal instability analysis to gas that is not in thermal equilibrium. Schwarz et al. (1972) assume that the unperturbed density is constant, Koyama \& Inutsuka (2000) that the unperturbed pressure is constant and Balbus (1986) consider a general unperturbed state. Balbus (1986) and Schwarz et al. (1972) go somewhat further than Koyama \& Inutsuka (2000) in that they use a JWKB approximation to take account of the time variation of the unperturbed state. However, in all cases the analysis is local i.e. only valid in the short wavelength limit.

Koyama \& Inutsuka (2000) assume that the gas is contracting uniformly so that lengths scale like $R(t)$. They introduce a scaled coordinate

$\bar{x}=\frac{x}{R}$

with $R(0)=1$. The primitive equations, (5), become

$\partial_{t} \mathbf{p}+\frac{1}{R} A \partial_{\bar{x}} \mathbf{p}=\frac{1}{R^{2}} \partial_{\bar{x}} \mathbf{c}+\mathbf{s}$,

with

$\mathbf{c}=\left[0,0,(\gamma-1) \kappa \partial_{\bar{x}} T\right]^{t}$,

and

$\mathbf{s}=\left[-\frac{\dot{R}}{R} \rho,-\ddot{R} \bar{x}-\frac{\dot{R}}{R} v,-\frac{\dot{R}}{R} \gamma p-(\gamma-1) \rho L\right]^{t}$.
The velocity, $v$, in $\mathbf{s}$ and $A$ is now the velocity in the comoving frame $v \rightarrow v-\dot{R} \bar{x}$.

They then consider a spatially uniform unperturbed state, $\mathbf{p}_{0}(t)$, with constant pressure, $p_{0}$, and zero velocity in the contracting frame. This satisfies

$p_{0}(t)=p_{0}(0), \quad \rho_{0}(t)=\frac{\rho_{0}(0)}{R(t)}, \quad v(t)=0, \frac{\dot{R}}{R}=-\frac{(\gamma-1)}{\gamma} \frac{\rho_{0} L}{p_{0}}$.

Integrating the last of these equations gives $R(t)$ and hence the solution. Note that this is only valid for regions small enough for the term $\ddot{R} \bar{x}$ to be negligible, which requires short wavelengths.

We assume a perturbation of the form

$$
\begin{aligned}
\rho(x, t) & =\rho_{0}(t)\left[1+\rho_{1} \exp (i \omega t-i k \bar{x})\right], \\
p(x, t) & =p_{0}\left[1+p_{1} \exp (i \omega t-i k \bar{x})\right], \\
v(x, t) & =v_{1} \exp (i \omega t-i k \bar{x}),
\end{aligned}
$$

where $\rho_{1}, p_{1}$ and $v_{1}$ are constants, which is equivalent to that used by Koyama \& Inutsuka (2000). Putting this into (45), linearising and neglecting $\ddot{R} \bar{x}$ gives

$i \omega \mathbf{p}_{1}-i \frac{k}{R} \mathrm{~A}_{c} \mathbf{p}_{1}=\mathrm{D}_{c} \mathbf{p}_{1}-\frac{k^{2}}{R^{2}} \mathrm{C}_{c} \mathbf{p}_{1}$,

where $\mathbf{p}_{1}=\left(\rho_{1}, v_{1}, p_{1}\right)^{t}$,

$\mathrm{A}_{c}=\left(\begin{array}{ccc}0 & 1 & 0 \\ 0 & 0 & p_{0} / \rho_{0} \\ 0 & \gamma & 0\end{array}\right)$

$\mathrm{D}_{c}=\left(\begin{array}{ccc}0 & 0 & 0 \\ 0 & -\sigma_{c} & 0 \\ \left(-\rho_{0} G-\rho_{0}^{2} G_{\rho}+p_{0} G_{T}\right) / p_{0} & 0 & -\gamma \sigma_{c}-G_{T}\end{array}\right)$,

and

$\mathrm{C}_{c}=\left(\begin{array}{ccc}0 & 0 & 0 \\ 0 & 0 & 0 \\ -\kappa^{\prime} / \rho_{0} & 0 & \kappa^{\prime} / \rho_{0}\end{array}\right)$.

Here

$G=(\gamma-1) L$

and

$\sigma_{c}=\frac{\dot{R}}{R}$

We can set $R=1$ since we are only interested in the stability of the original state. The dispersion relation is then

$\left|\omega \mathrm{I}-k \mathrm{~A}_{c}+i \mathrm{D}_{c}-k^{2} i \mathrm{C}_{c}\right|=0$,

which we can write as

$P=P_{r}-i P_{i}$.

In the previous subsections we showed that it is useful to split the dispersion relation into polynomials associated with the different physical processes, the adiabatic system, the energy source and thermal conduction. There are now four 
different processes: adiabatic, energy source, thermal conduction and the source due to the isobaric contraction.

We therefore write equation (57) as

$P_{r}=P_{0}-\frac{1}{k^{2}}\left(P_{13}+P_{33}\right)-P_{23}, \quad P_{i}=\frac{1}{k}\left(P_{1}+P_{3}\right)+k P_{2}$,

with $P_{0}$, and $P_{2}$ given by equations (15) and (30) and

$$
\begin{aligned}
& P_{1}=G_{T}\left(\mu^{2}-a_{e}^{2}\right)+G, \quad P_{3}=\sigma_{c}(\gamma+1) \mu^{2}, \\
& P_{13}=\sigma_{c} G_{T} \mu, \quad P_{23}=\frac{\sigma_{c} \kappa}{\rho_{0}} \mu, \quad P_{33}=\gamma \sigma_{c}^{2} \mu .
\end{aligned}
$$

Here the suffices 1, 2, 3 are associated with the energy source, thermal conduction and the isobaric contraction respectively. The dispersion relation is split into auxiliary polynomials $P_{1}, P_{2}, P_{3}$ due to each process in isolation, $P_{13}, P_{23}$ due to interactions between them and $P_{33}$ due to selfinteraction of the isobaric contraction. Note that the isobaric contraction has a self-interaction because it affects both the velocity and pressure equation, whereas the other processes only appear in the pressure equation.

If we ignore conduction, then for the condensation mode at large $k$ we get

$\omega=i G_{T} \frac{\left(a_{e}^{2}-G / G_{T}\right)}{a_{f}^{2}}+O\left(1 / k^{2}\right)$

This clearly also applies to the magnetic case if the field is not exactly perpendicular. It is a reasonable approximation to the growth rate of the most unstable short wavelength mode whenever the Field length is significantly smaller than the acoustic wavelength. We therefore have short wavelength instability when

$a_{e}^{2} G_{T}-G<0$,

which is just the isobaric instability condition for nonequilibrium states given by Balbus (1986).

In order to determine when (60) is a good approximation to the maximum growth rate, we need the Field length for this case. The coefficient of $\mu^{2}$ in $P_{i}$ is

$\frac{1}{k}\left[G_{T}+\sigma_{c}(\gamma+1)\right]+\frac{k \kappa^{\prime}}{\rho_{0}}$,

and this must be positive for stability. $P_{i}$ has real roots if

$k^{2}>\frac{\rho_{0}}{\kappa^{\prime} c_{T}^{2}}\left(G-G_{T} a_{e}^{2}\right) G_{T}$.

In combination, (62) and (63) tell us that we have stability if

$k>k_{f}=\left[\frac{\rho_{0}}{\kappa^{\prime}} \max \left\{-G_{T}-\sigma_{c}(\gamma+1), \frac{\left(G-G_{T} a_{e}^{2}\right)}{c_{T}^{2}}\right\}\right]^{1 / 2}$

This replaces the expression (32) for the Field wavenumber. It is everywhere much larger than the thermal wavenumber for any plausible form of $L$, such as the one considered in the next section.

Although Koyama \& Inutsuka (2000) only considered an isobaric unperturbed state, their analysis is valid for short wavelengths and any unperturbed state, provided the growth rate is large compared to $\left|\sigma_{c}\right|=|\dot{R} / R|$. In particular, for the isochoric state considered by Schwarz et al. (1972), the equations are the same with $\sigma_{c}=0, R=1, p_{0}=p_{0}(t)$ and $\rho_{0}=$ const. Since (60) is independent of $\sigma_{c}$, the condition (61) also applies in this case.

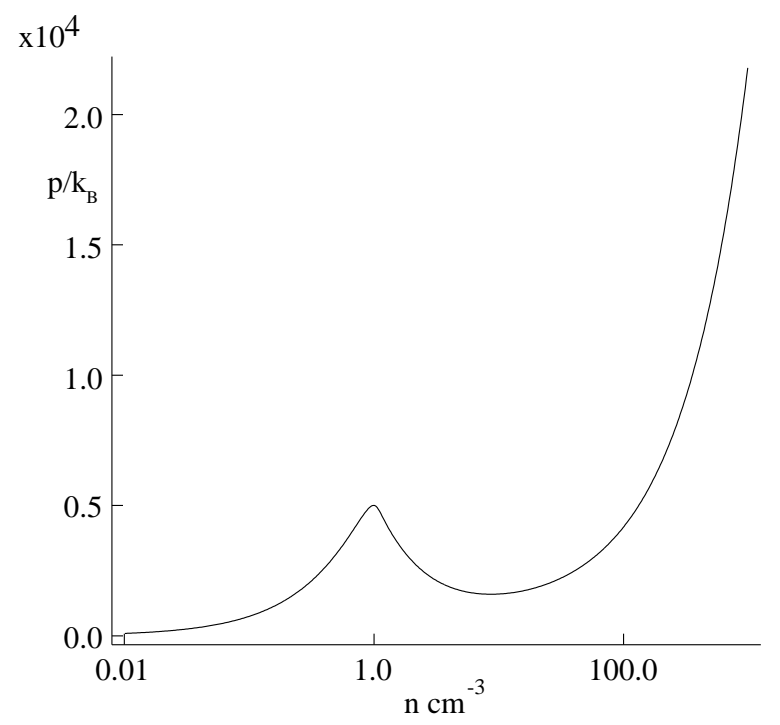

Figure 1. The pressure divided by the Boltzmann constant, $k_{B}$, on the equilibrium curve as a function of particle density.

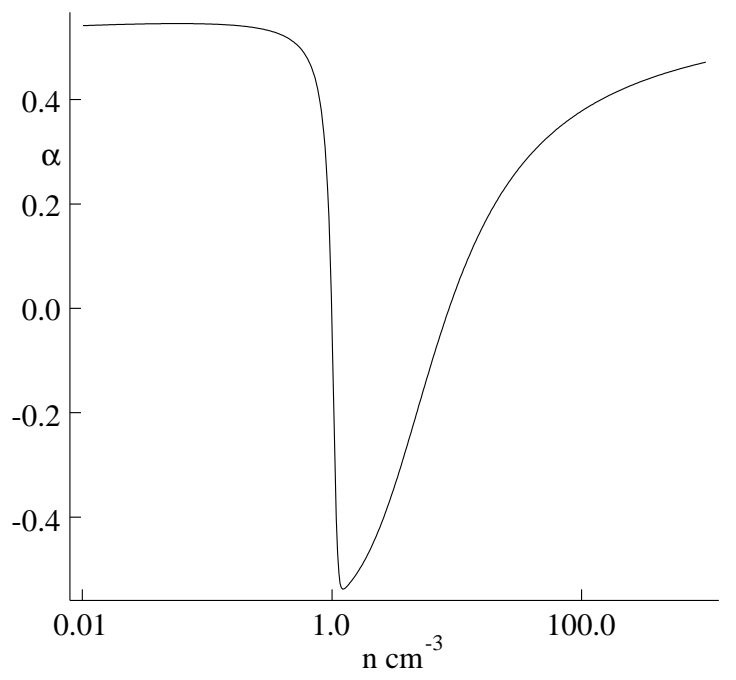

Figure 2. The dimensionless parameter, $\alpha$, defined by equation (21) as a function of particle density.

\section{KOYAMA \& INUTSUKA ENERGY SOURCE}

Koyama \& Inutsuka (2002) used a thermal energy loss function of the form

$L(\rho, T)=\frac{\rho}{m_{H}^{2}} \Lambda(T)-\frac{1}{m_{H}} \Gamma$,

where $T$ is in Kelvin, $\Gamma=210^{-26} \mathrm{erg} \mathrm{s}^{-1}$ and

$\bar{\Gamma}=10^{7} \exp \left(\frac{-1.184 \times 10^{5}}{T+1000}\right)+1.410^{-2} T^{1 / 2} \exp \left(\frac{-92}{T}\right)$,

This has been used by a number of authors (e.g. Vázquez- 


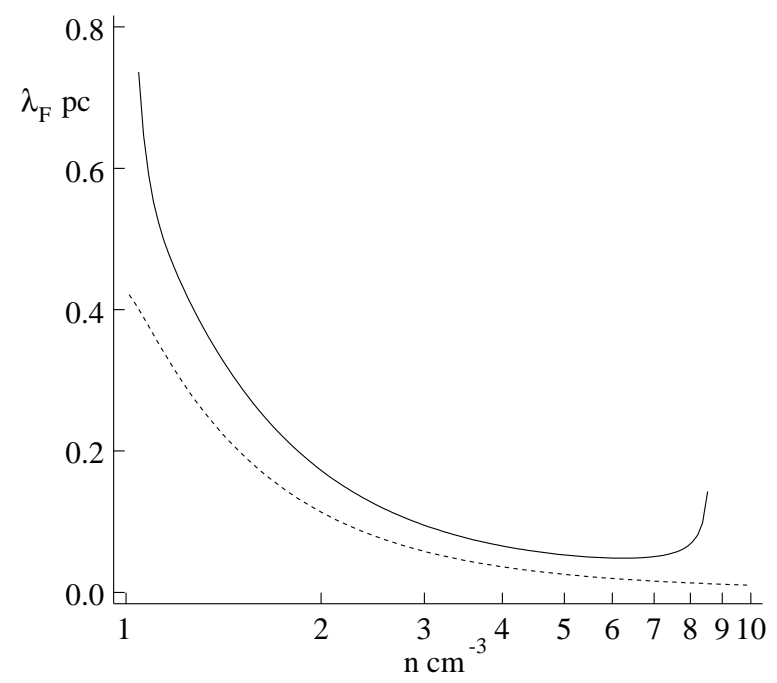

Figure 3. The Field length as a function of particle density from (33) (solid line) and from (34) (dashed line).

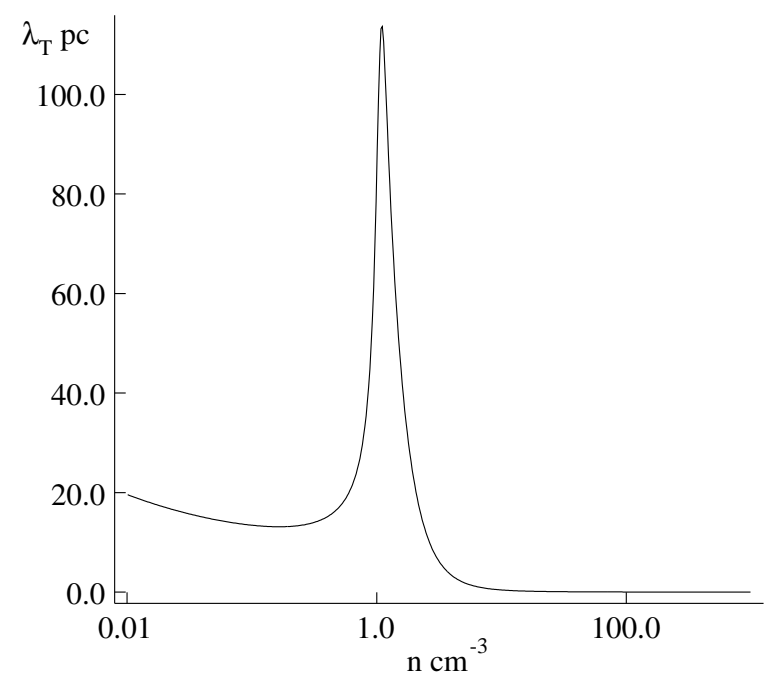

Figure 4. The thermal length as a function of particle density from (18).

Semadini et al. 2007; Kim et al. 2008; Wareing et al. 2016a,b, 2017, 2018, 2019).

Koyama \& Inutsuka (2004) include a thermal conductivity

$\kappa=2.510^{3} T^{1 / 2}$,

which is appropriate for $T<4.510^{4} K$ (Parker 1953). The kinematic viscosity is given by

$v=\frac{(\gamma-1)}{\gamma} \frac{m}{k_{B}} \frac{\kappa}{\rho} P_{r}$,

where $P_{r}$ is the Prandtl number, which is $2 / 3$ for a monotomic gas. (a)

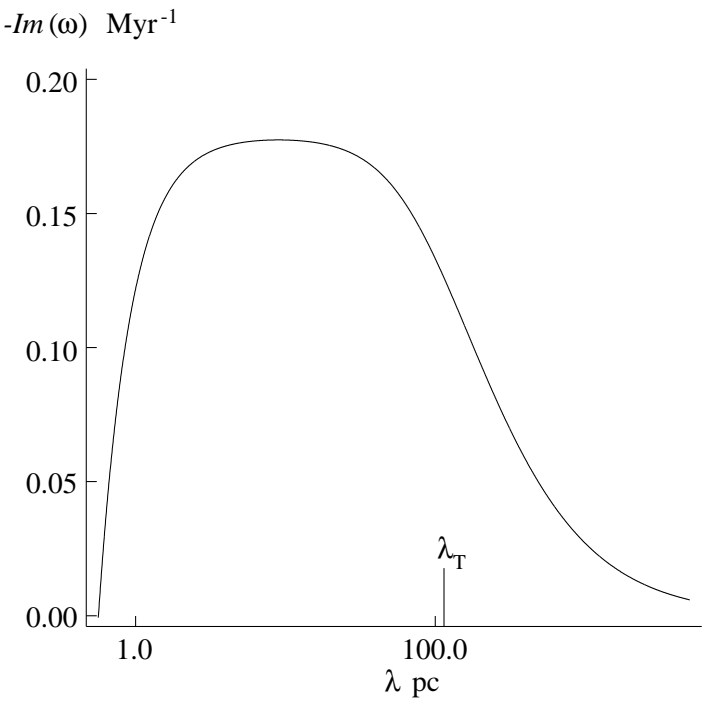

(b) $-\operatorname{Im}(\omega) \mathrm{Myr}^{-1}$

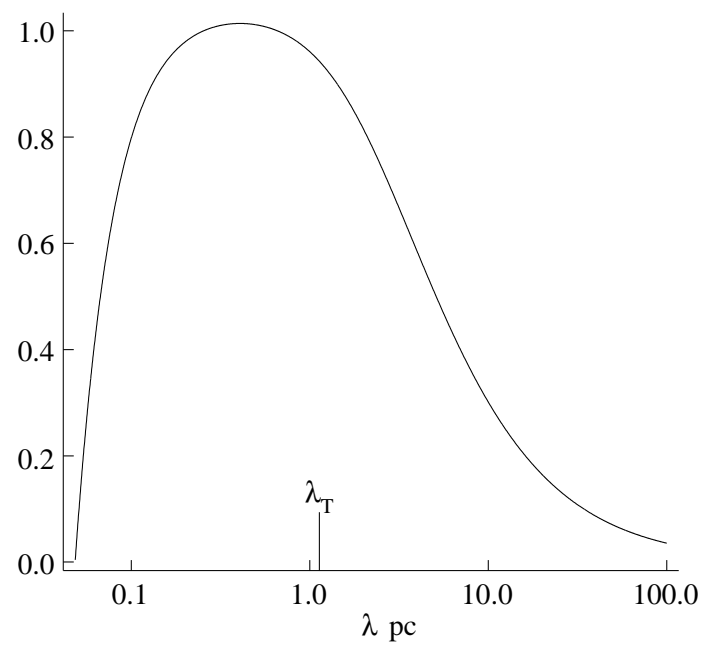

Figure 5. The growth rate of the condensation mode as a function of wavelength. a) $n=1.1$, b) $n=6.2517$ (the density at which the Field length is a minimum).

\subsection{Equilibrium states}

The unstable temperature and density ranges for the isobaric condensation mode are $184 \mathrm{~K} \leq T \leq 5039 \mathrm{~K}$ and $0.9936 \leq n \leq 8.6818$. The equilibrium pressure is shown in Fig. 1. From Fig. 2 we can see that $\alpha<1$ everywhere, so that equation (22) tells us that the isentropic modes are always stable. Fig. 3 shows the Field length from equations (33) and (34) in the unstable region, from which it can be seen that they are indeed very different. We can see from Fig. 4 that the thermal wavelength is rather large in most of the unstable region, varying between the typical size of giant molecular clouds and that of the translucent clumps. Although Fig. 5 shows that the growth rate of the condensation mode as a function of wavelength does have a maximum, 


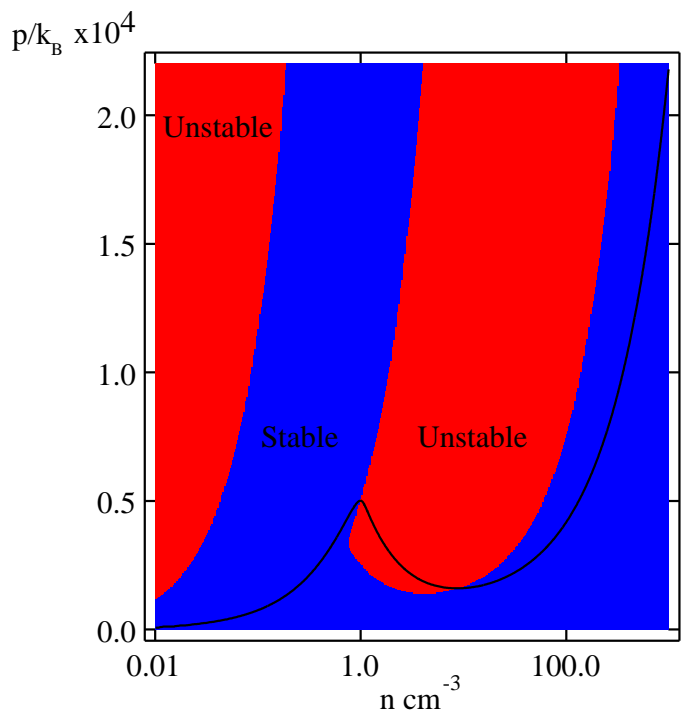

Figure 6. The unstable region in the $n-p$ plane according to the Balbus criterion (61). The line is the equilibrium curve

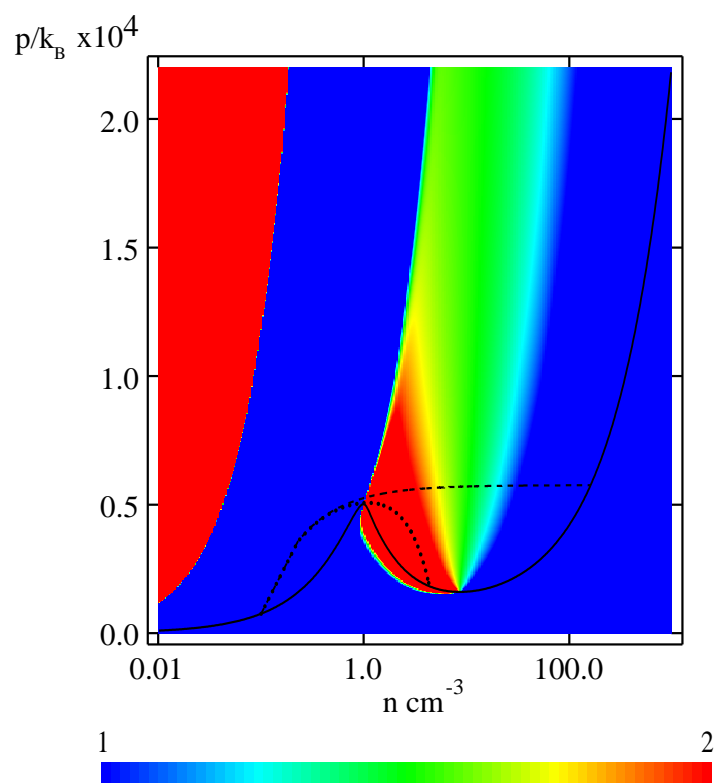

Figure 7. Linear plot of the ratio of the maximum growth rate given by (60) to $\left|\sigma_{c}\right|$. The solid line is the equilibrium curve. The dashed line is the track of gas passing through a Mach 2 hydrodynamic shock propagating into gas in thermal equilibrium with $n=0.1$. The dotted line is the track for an oblique MHD fast shock with a thermal Mach number of 2 propagating into an equilibrium state with $n=0.1$, equal parallel and perpendicular fields and plasma $\beta=200$ (shock 2 in Table 1 ).

it is not so sharp that one particular wavelength is strongly favoured.

\subsection{Non-equilibrium states}

A number of authors have considered instability occurring behind shocks generated by colliding flows that drive the gas into an unstable non-equilibrium state (e.g. Hennebelle \& Pérault 2000; Koyama \& Inutsuka 2000, 2002; Heitsch et al. 2009; Inoue \& Inutsuka 2008, 2009; Fogerty et al. 2016). Fig. 6 shows the region in the $n-p$ plane in which (61) indicates instability and agrees with fig. 4 in Inoue \& Inutsuka (2008). Note that the unstable region at low densities is unphysical since it corresponds to temperatures above $10^{4} \mathrm{~K}$, for which the energy source function (65) is not valid. A more realistic model of the interstellar cooling curve above $10^{4} \mathrm{~K}$, such as that in Gnat \& Ferland (2012), gives isochoric instability for $T>10^{5} \mathrm{~K}$.

Koyama \& Inutsuka (2000) applied the analysis in Section 2.4 to thermally unstable gas cooling behind a shock, but it is only valid when the growth rate given by (60) is large compared to the rate of contraction, $\left|\sigma_{c}\right|=|\dot{R} / R|$. Gas cooling behind shocks that lead to a phase change must indeed pass through the unstable region above the equilibrium curve, but Fig. 7 shows that the maximum growth rate is not large compared to $\left|\sigma_{c}\right|$ in most of this region. In fact the analysis only gives reasonable quantitative results for shocks that are not much stronger than that required to trigger a transition to the cold phase.

\subsection{Steady shocks}

Fig. 7 also shows the path in the $n-p$ plane for a steady shock and Fig. 8 the structure of its cooling region. It can be seen from Fig. 8b that the flow is indeed approximately isobaric in the unstable region. From Fig. $8 \mathrm{~d}$ we can see that it is unstable for $-29.934<x<-13.742$, but that $-\mathfrak{I}(\omega) /\left|\sigma_{c}\right|<5.5$. The analysis is therefore only marginally valid even for a shock such as this that only just triggers a phase change. Note that the cooling lengths for this shock and the magnetic version shown in Fig. 9 are very large because the path in the $n-p$ plane passes very close to the equilibrium curve at $n=1$ where the cooling time is long.

The effect of pure thermal instability in shocks that drive the gas to the cold stable state is not very interesting. Although the gas must pass through the unstable region in which the instability can grow, we will see this does not have a dramatic effect on the overall structure of cooling region. Since the gas ends up in a stable region in which a two-phase medium is impossible, any density inhomogeneities generated by the instability must decay. Colliding flows that lead to such shocks do produce interesting density structures, but these are due to other effects such as the thin-shell instability, Rayleigh-Taylor instability or self-gravity (e.g. Koyama \& Inutsuka 2002; Heitsch et al. 2008a,b; Fogerty et al. 2016)

Thermal instability can only produce persistent density variations if the gas remains in the unstable region as it cools towards the equilibrium state, which cannot happen behind a shock unless there is a magnetic field. Fig. 7 also shows the path of an oblique MHD fast shock, from which we can see that even a small initial magnetic field can lead to a final state on the unstable part of the equilibrium curve. This is because there is enough compression to increase the magnetic field to the point where the magnetic pressure dominates, as can be seen from Fig. 9b. Fig. 9d also tells us that 
(a)

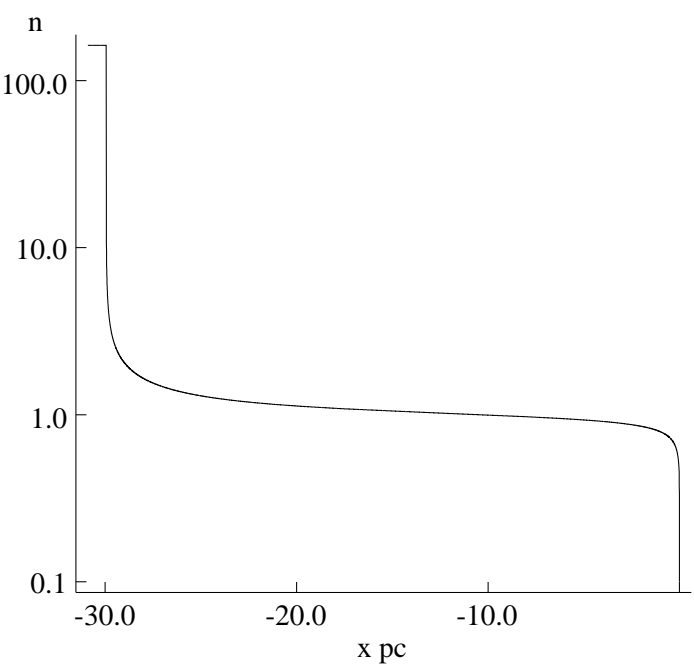

(c)

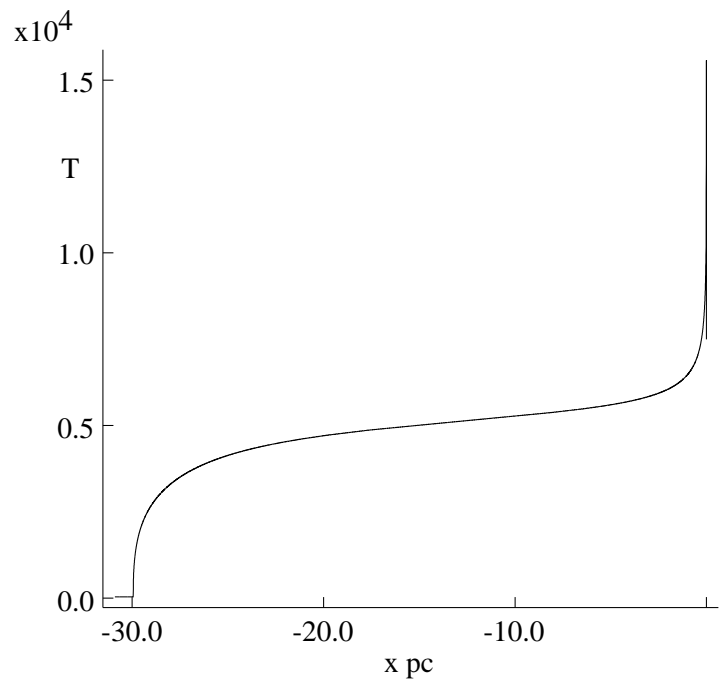

(b)

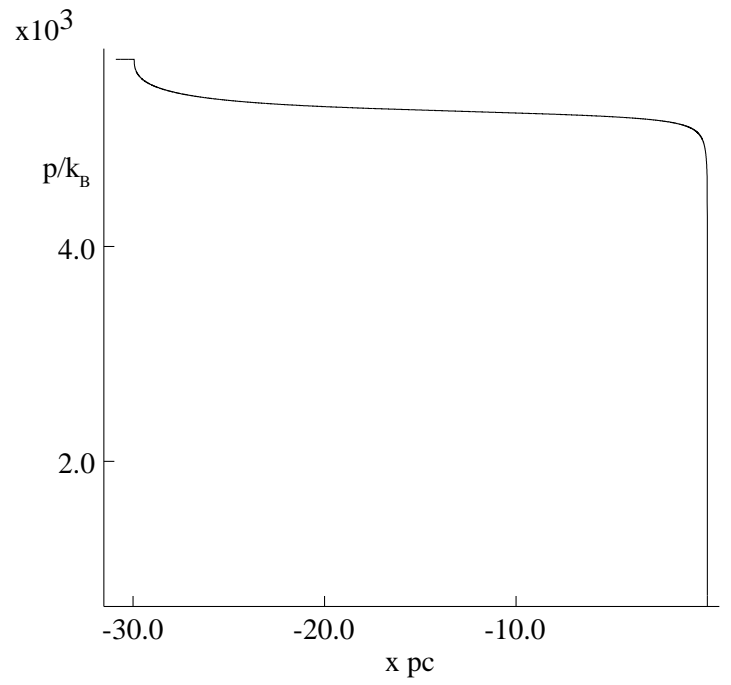

(d)

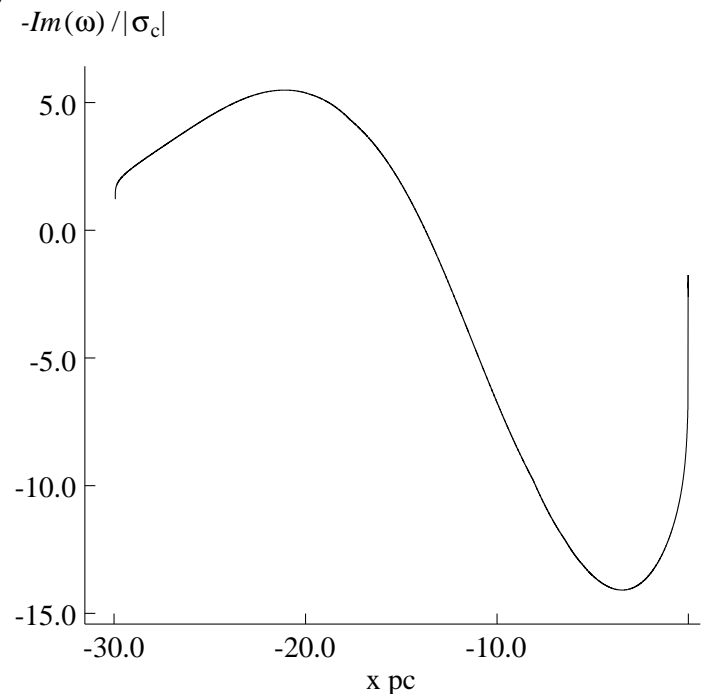

Figure 8. The solution for a steady Mach 2 hydrodynamic shock propagating into gas in thermal equilibrium with $n=0.1$. a) density, b) pressure, c) temperature, d) the ratio of the maximum growth rate given by (60) to $\left|\sigma_{c}\right|$. Note that this is infinite in the final state since $\left|\sigma_{c}\right|=0$ there.

it is unstable for $x<-37.4$ and the linear analysis is reasonably accurate since the growth rate is significantly larger than $\left|\sigma_{c}\right|$. Note that the growth rate is positive in the final state, unlike the shock with a stable final state shown in Fig. 8.

Such steady shock solutions in which the gas reaches the unstable part of the equilibrium curve will obviously not occur in nature and indeed simulations (e.g. Koyama \& Inutsuka 2002; Audit \& Hennebelle 2005) show that they are unstable. The only possibility is that the gas separates into stable warm and cold phases if the gas pressure is in the range for which these phases can coexist (Inoue \& Inutsuka 2009). This also happens if one perturbs gas on the unstable part of the equilibrium curve (e.g. Wareing et al. 2019). The most interesting shocks are therefore those for which the density in the final state is in the unstable region of the equilibrium curve. The largest density contrast between the phases occurs when the density in the final state is near the lower end of the unstable region i.e. $n \simeq 1$.

The steady shock solutions are described by four parameters: the upstream density, Mach number, plasma $\beta$ and the angle between the magnetic field and the shock normal. There is therefore a three-fold infinity of solutions that can reach any given point on the equilibrium curve. Table 1 gives the properties of a number of such solutions, for which all of the MHD shocks end up on the unstable part of the equilibrium curve. Of these, we expect shock 5 to give the largest density contrast between the phases since its final density is closest to the lower stability limit. 
(a)

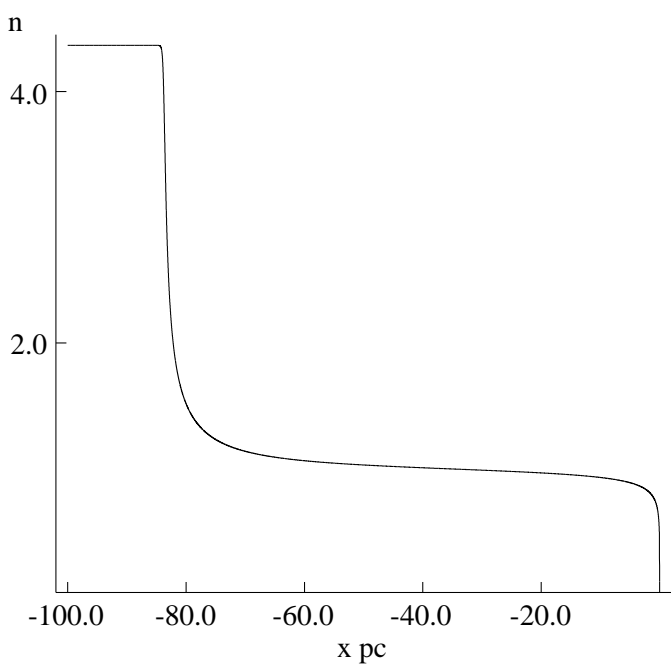

(c)

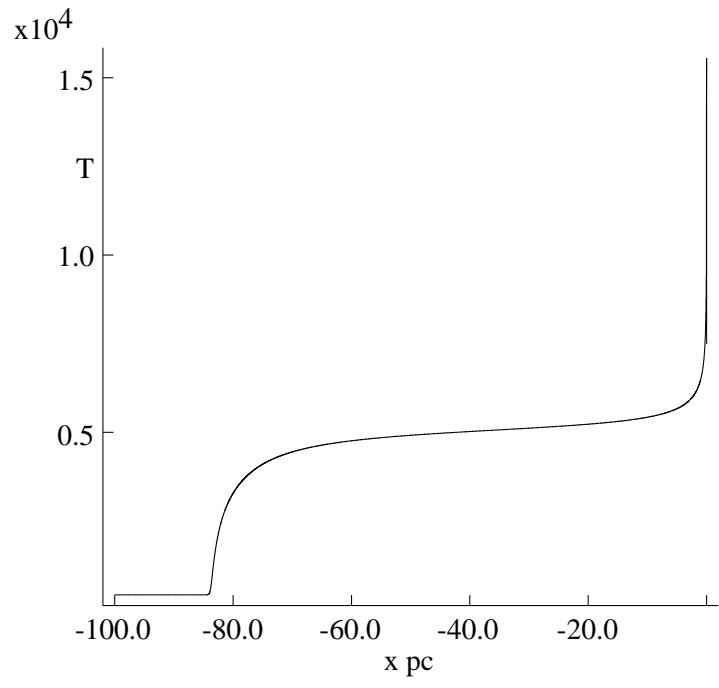

(b)

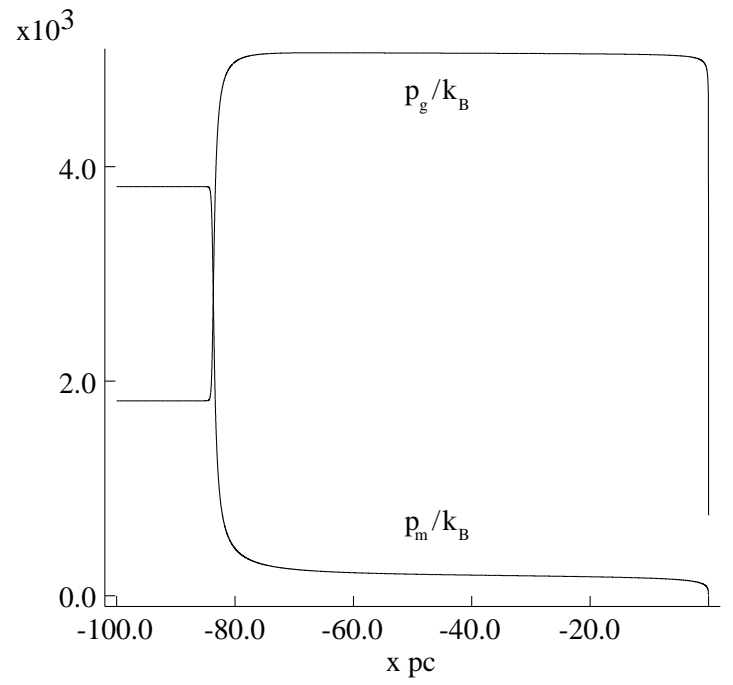

(d)

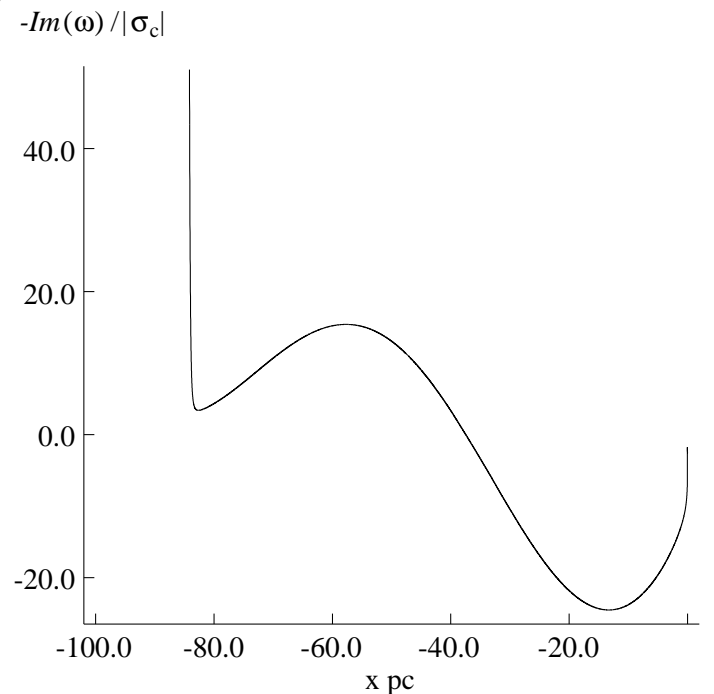

Figure 9. The solution for a steady oblique MHD fast shock with a thermal Mach number of 2 propagating into an equilibrium state with $n=0.1, B_{x}=B_{y}$ and plasma $\beta=200$ (shock 2 in Table 1). a) density, b) gas and magnetic pressure, c) temperature, d) the ratio of the maximum growth rate given by (60) to $\left|\sigma_{c}\right|$. Note that this is infinite in the final state since $\left|\sigma_{c}\right|=0$ there.

The table also shows the amplification factor of the instability defined by

$A=\exp \left\{\int \max [0,-\mathfrak{I}(\omega)] \frac{\mathrm{d} x}{v_{x}}\right\}$,

where the integral is from the shock to the intersection with the equilibrium curve. This definition excludes the damping in the stable regions, which means that the amplification factor for the purely hydrodynamic shocks is seriously overestimated.

The cooling region in both the hydrodynamic (shock 1 ) and MHD (shock 2) shock is very long because the track in the $n-p$ plane passes close to the equilibrium curve, but Table 1 shows that this is exceptional. Stronger shocks have shorter cooling regions because their track is far from the equilibrium curve and MHD shocks have lower densities and hence longer cooling regions than the corresponding hydrodynamic ones. It is also clear from the difference between the final density in the hydrodynamic and MHD cases, that it does not require much of an upstream magnetic field for the magnetic pressure to dominate in the final state.

The fact that even a very small magnetic field has such a large effect, means that purely hydrodynamic simulations are of little relevance. This is pretty obvious and has been pointed out by several authors (e.g. Hennebelle \& Pérault 2000; Heitsch et al. 2009; Inoue \& Inutsuka 2008, 2009). They all conclude that the immediate formation of very dense gas in colliding flows is prevented by a typical magnetic field unless it is very closely aligned with the flow. Table 1 confirms this: the magnetic field dominates in the final state for shocks $2,4,5$ and 7 even though the initial magnetic field is implausibly small. It also dominates for the 
(a)

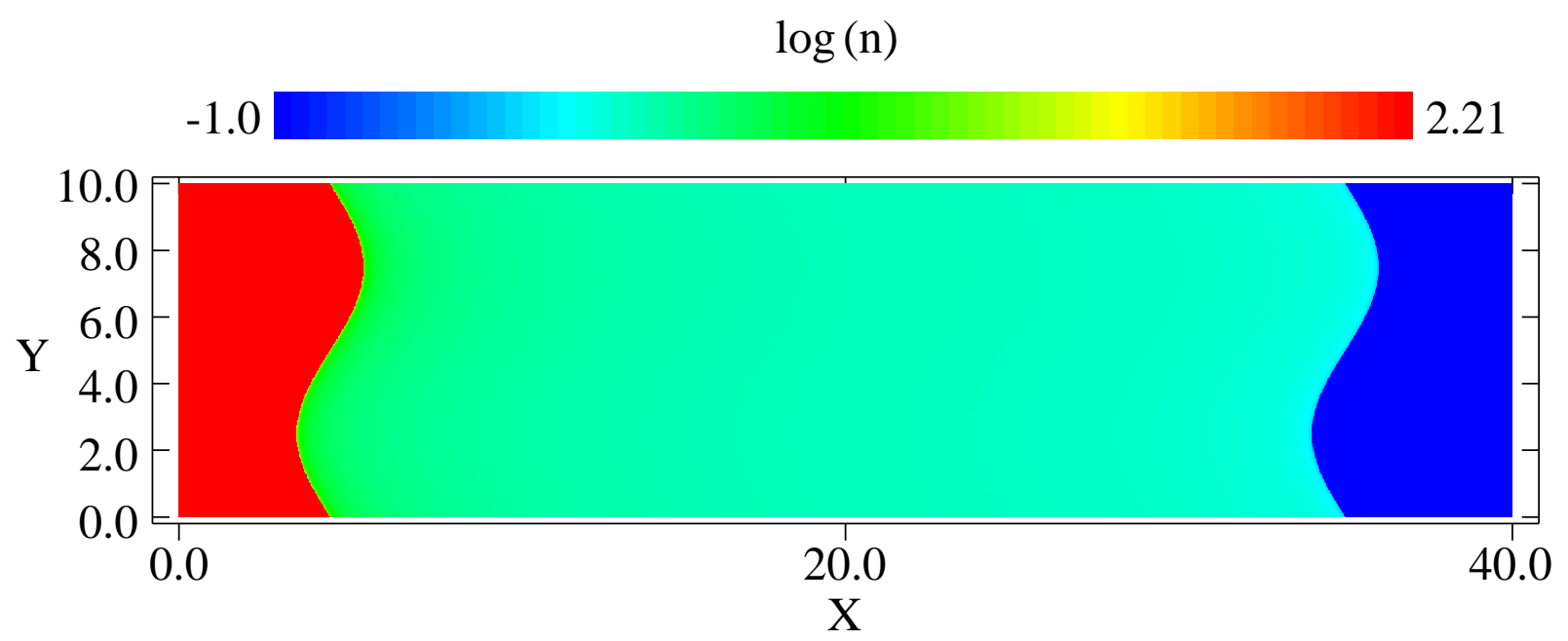

(b)

$\log (\mathrm{n})$

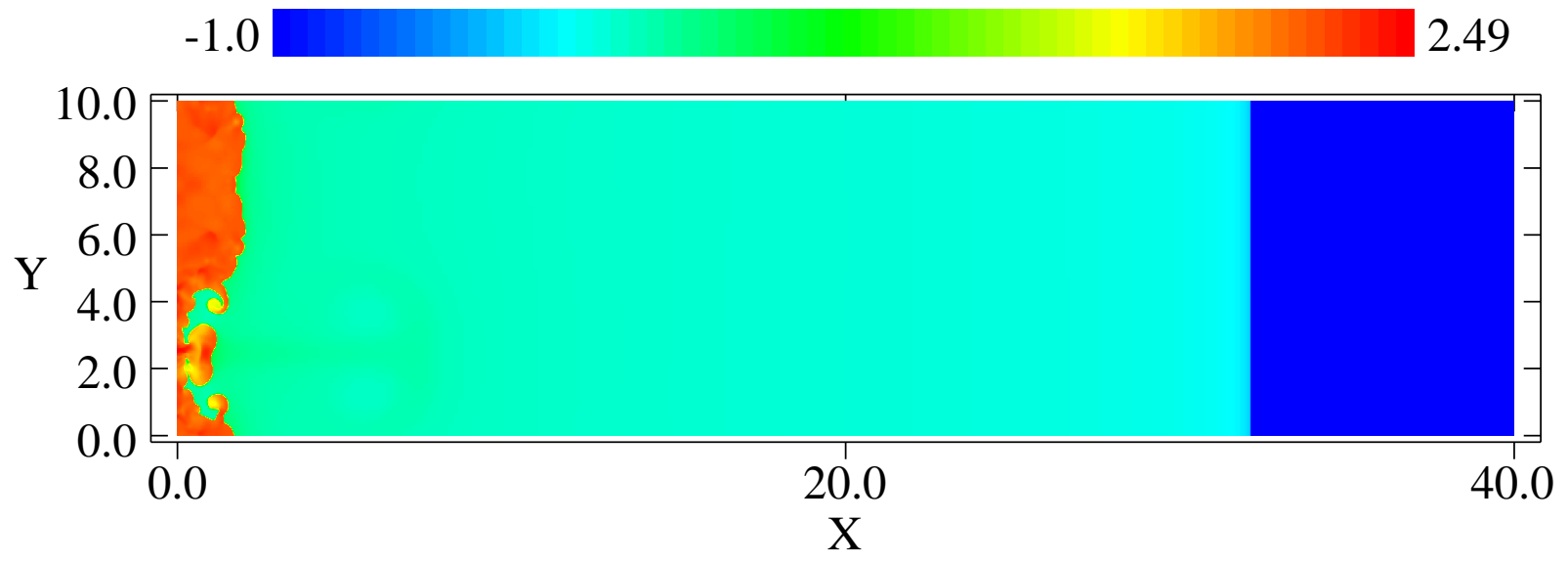

(c)

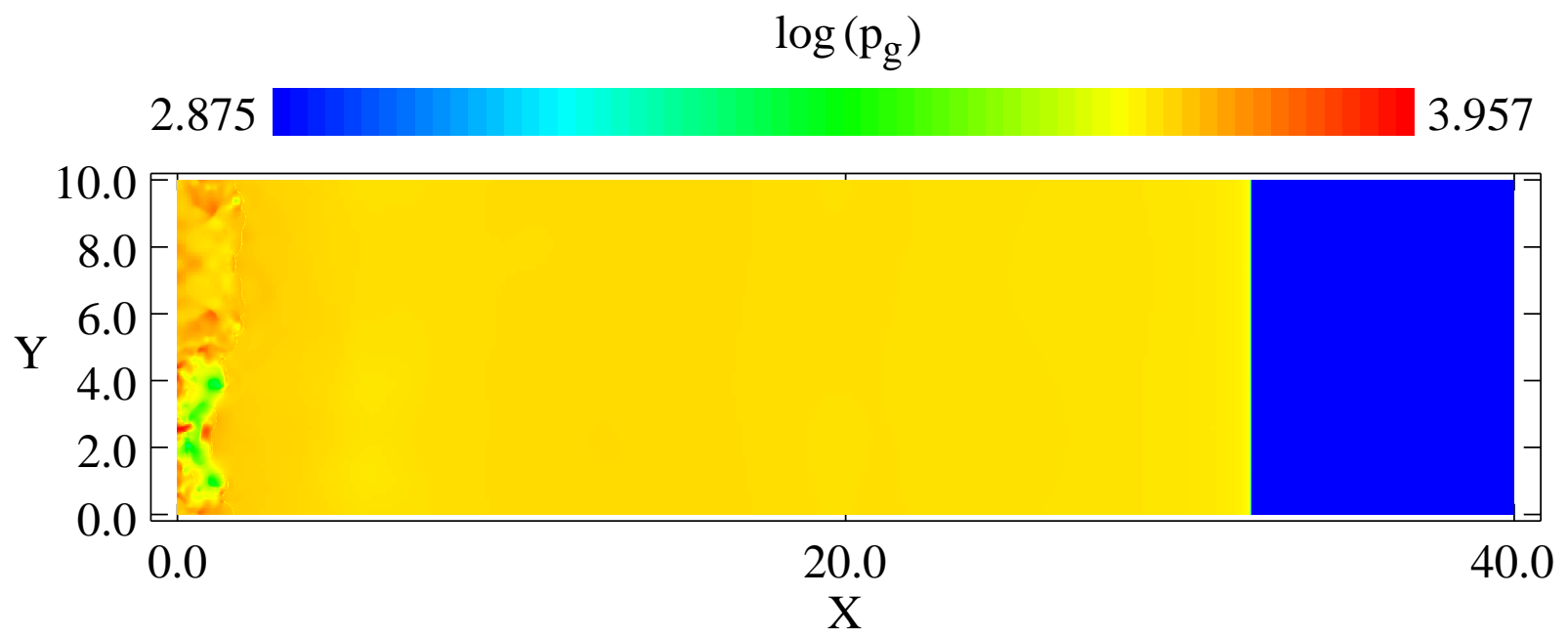

Figure 10. Log density for the perturbed 2D hydrodynamic shock 1. (a) Initial state, (b) at $t=20$ Myrs. (c) Log pressure at $t=20$ Myrs. There were 5 grid levels with a finest grid spacing of 0.02 pc. Distances are in pc. 
(a)

$\log (\mathrm{n})$
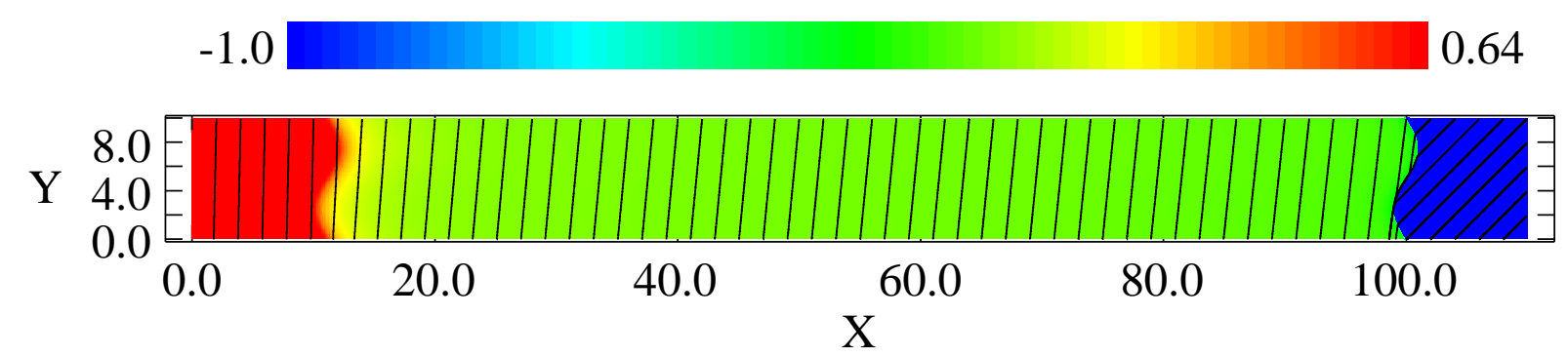

(b)

\section{$\log (\mathrm{n})$}
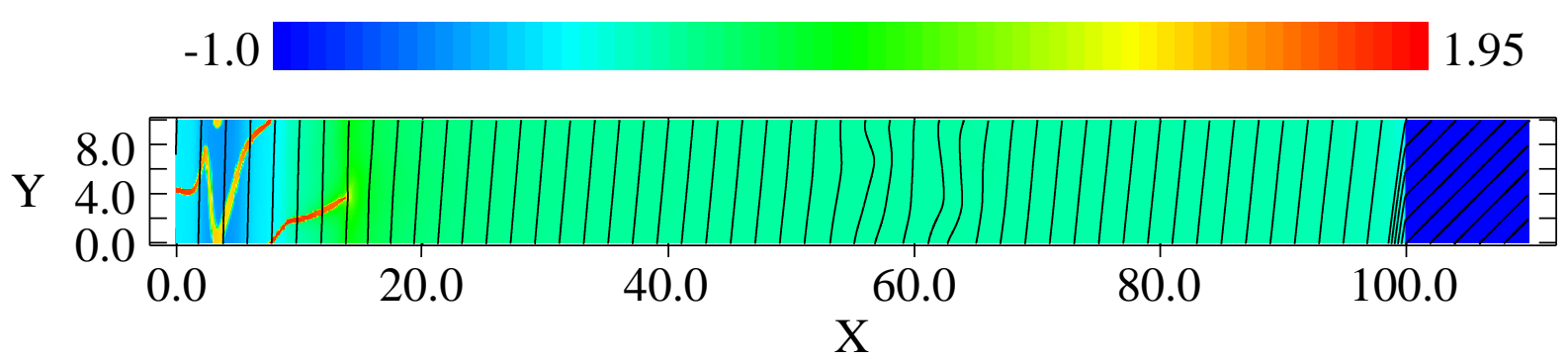

(c)

$$
\log \left(\mathrm{p}_{\mathrm{g}}\right)
$$
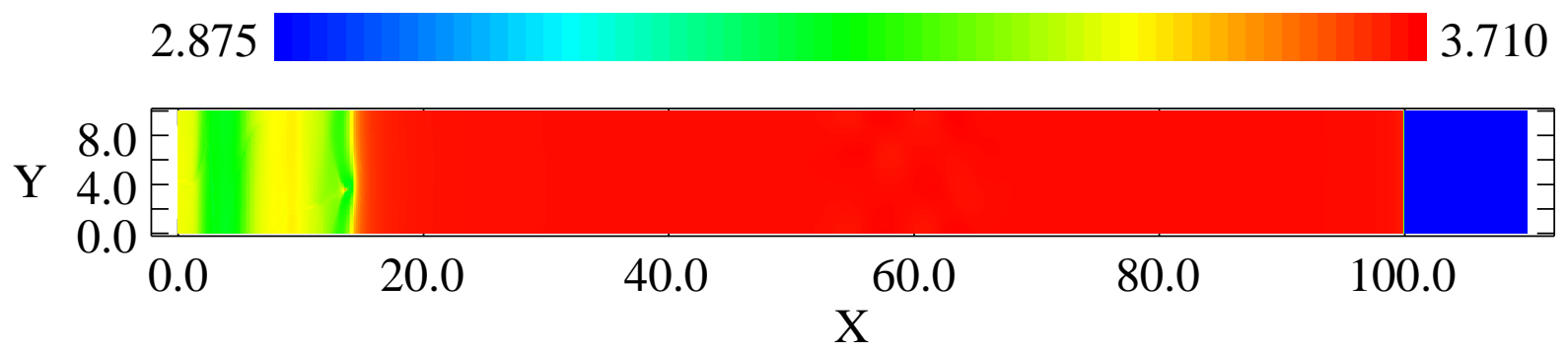

Figure 11. Log density and magnetic field lines for the perturbed 2D MHD fast shock 2 (a) Initial state, (b) at $t=20$ Myrs. (c) Log pressure at $t=20 \mathrm{Myrs}$. There were 5 grid levels with a finest grid spacing of $0.02 \mathrm{pc}$. Distances are in pc. The FWHM width of the filaments is $\simeq 0.52 \mathrm{pc}$.

Table 1. Properties of steady shock solutions: $M_{T h}$ - thermal Mach number; $\theta$ - angle between field and shock perpendicular; $n$ - particle number density; $\beta$ - plasma $\beta ; B$ - magnitude of the magnetic field; $l_{c}$ - length of cooling region; $A$ - amplification factor given by (69). The suffices $u$ and $f$ denote the upstream and final values. Shock 10 is the perpendicular shock generated by the collision in Inoue \& Inutsuka (2008) and shock 11 the case $1 \mathrm{a} 15^{\circ}$ shock in Inoue \& Inutsuka (2008)

\begin{tabular}{llllllllllll} 
& \multicolumn{1}{c}{$M_{T h}$} & $\theta_{u}$ & $\theta_{f}$ & $n_{u}$ & $n_{f}$ & $\beta_{u}$ & \multicolumn{1}{c}{$\beta_{f}$} & $B_{u} \mu \mathrm{G}$ & $B_{f} \mu \mathrm{G}$ & $l_{c} \mathrm{pc}$ & $\mathrm{A}$ \\
\hline \hline 1 & $2.0\left(18.56 \mathrm{~km} \mathrm{~s}^{-1}\right)$ & $\mathrm{NA}$ & $\mathrm{NA}$ & 0.1 & 162.5 & $\infty$ & $\infty$ & 0 & 0 & 30.49 & $3.510^{3}$ \\
2 & $2.0\left(18.56 \mathrm{~km} \mathrm{~s}^{-1}\right)$ & $45^{\circ}$ & $88.7^{\circ}$ & 0.1 & 4.368 & 200 & 0.4764 & 0.114 & 3.638 & 85.04 & $4.010^{4}$ \\
3 & $3.0\left(27.84 \mathrm{~km} \mathrm{~s}^{-1}\right)$ & $\mathrm{NA}$ & $\mathrm{NA}$ & 0.1 & 454.1 & $\infty$ & $\infty$ & 0 & 0 & 1.928 & $2.710^{2}$ \\
4 & $3.0\left(27.84 \mathrm{~km} \mathrm{~s}^{-1}\right)$ & $45^{\circ}$ & $89.2^{\circ}$ & 0.1 & 7.212 & 200 & 0.1578 & 0.114 & 5.954 & 2.782 & $9.310^{1}$ \\
5 & $3.0\left(27.84 \mathrm{~km} \mathrm{~s}^{-1}\right)$ & $45^{\circ}$ & $85.8^{\circ}$ & 0.1 & 1.243 & 10 & 0.6350 & 0.510 & 4.865 & 66.05 & $4.410^{2}$ \\
6 & $2.0\left(17.02 \mathrm{~km} \mathrm{~s}^{-1}\right)$ & $\mathrm{NA}$ & $\mathrm{NA}$ & 0.5 & 1126 & $\infty$ & $\infty$ & 0 & 0 & 1.336 & $2.710^{2}$ \\
7 & $2.0\left(17.02 \mathrm{~km} \mathrm{~s}^{-1}\right)$ & $45^{\circ}$ & $85.5^{\circ}$ & 0.5 & 5.615 & 12.5 & 0.0811 & 0.935 & 8.502 & 4.431 & $5.810^{1}$ \\
8 & $2.0\left(17.02 \mathrm{~km} \mathrm{~s}^{-1}\right)$ & $15^{\circ}$ & $63.2^{\circ}$ & 0.5 & 1.337 & 1 & 0.2716 & 3.305 & 7.283 & 52.00 & 7.6 \\
9 & $2.0\left(17.02 \mathrm{~km} \mathrm{~s}^{-1}\right)$ & $10^{\circ}$ & $64.0^{\circ}$ & 0.5 & 1.421 & 1 & 0.2532 & 3.305 & 7.423 & 37.84 & 6.05 \\
10 & $3.2\left(26.91 \mathrm{~km} \mathrm{~s}^{-1}\right)$ & $90^{\circ}$ & $90^{\circ}$ & 0.57 & 3.869 & 3.035 & 0.0436 & 2.000 & 13.61 & 7.275 & $4.610^{1}$ \\
11 & $3.1\left(25.74 \mathrm{~km} \mathrm{~s}^{-1}\right)$ & $15^{\circ}$ & $78.5^{\circ}$ & 0.67 & 5.335 & 1.542 & 0.0296 & 3.000 & 14.55 & 4.231 & $2.210^{1}$ \\
\hline
\end{tabular}


(a)

$\log (\mathrm{n})$
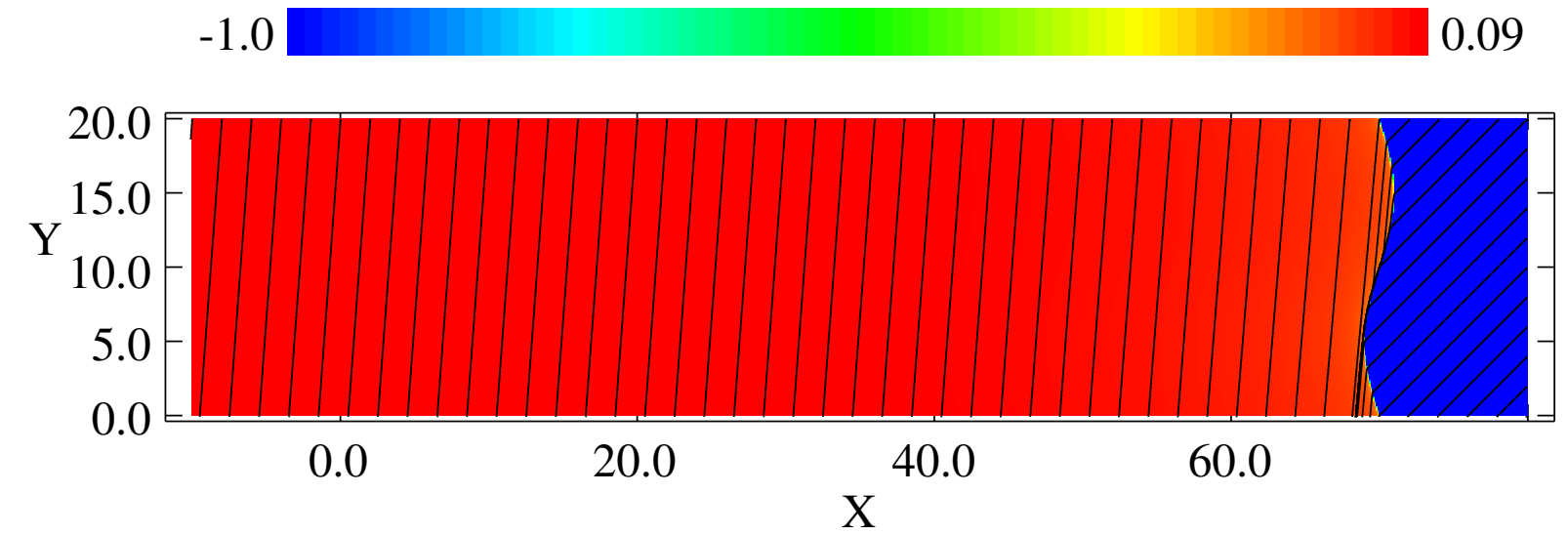

(b)

$\log (n)$

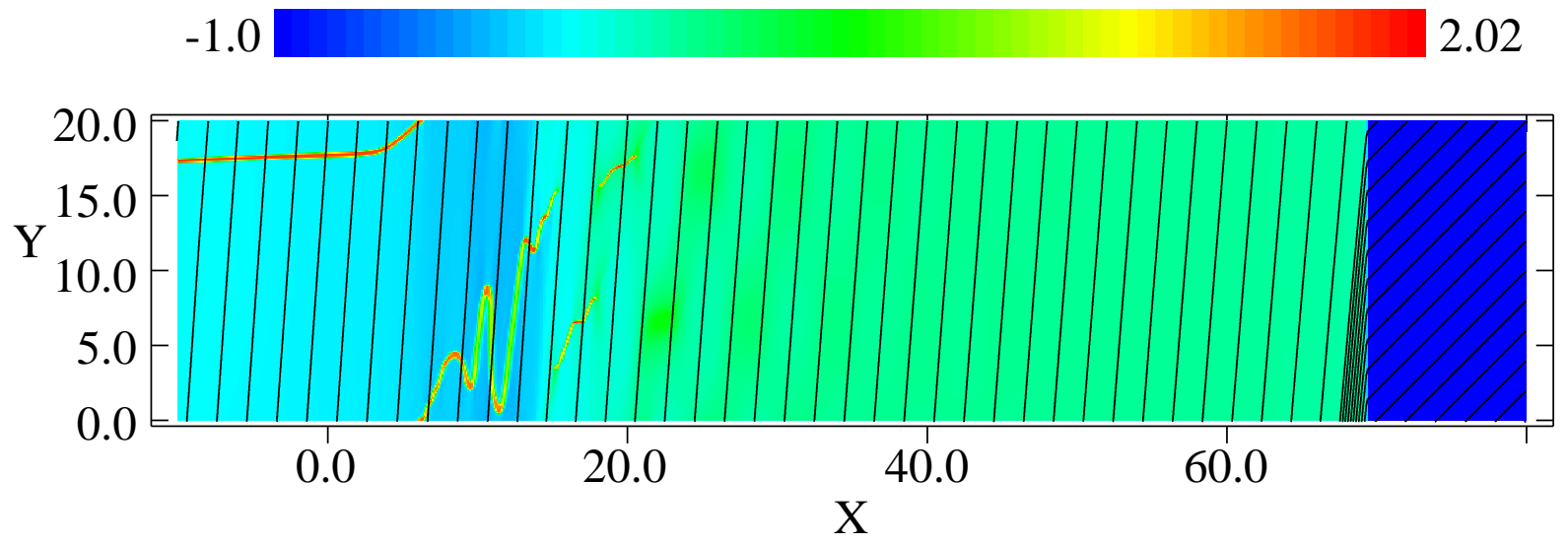

(c)

$$
\log \left(p_{g}\right)
$$

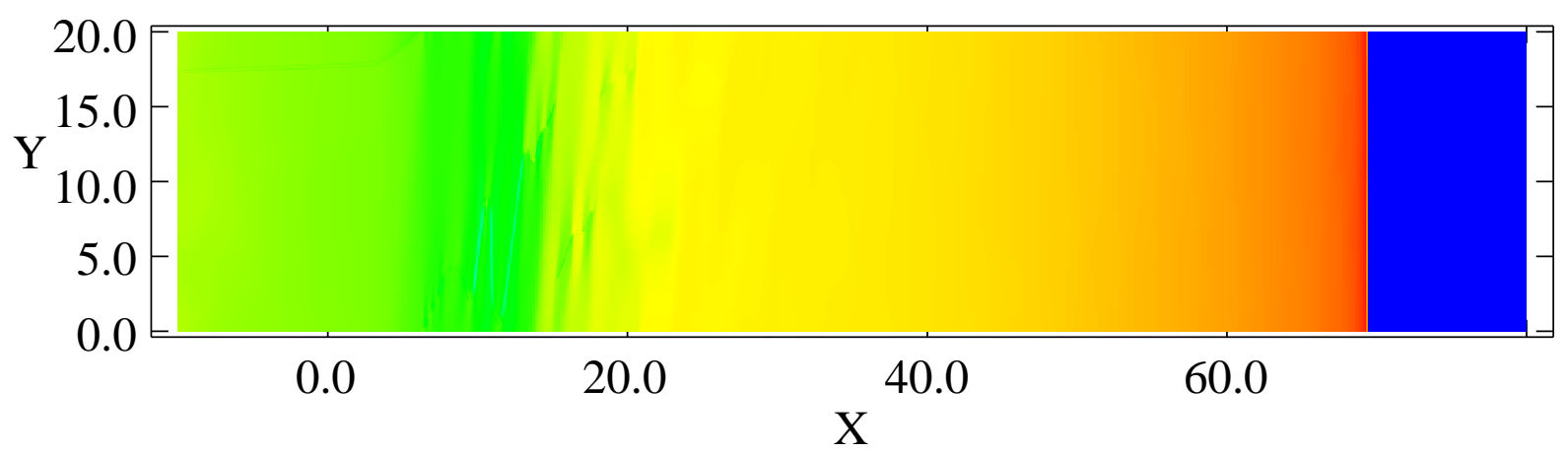

Figure 12. Log density and magnetic field lines for the perturbed 2D MHD fast shock 5 (a) Initial state, (b) at $t=25$ Myrs. (c) Log pressure at $t=25$ Myrs. There were 5 grid levels with a finest grid spacing of $0.02 \mathrm{pc}$. Distances are in pc. The FWHM width of the filaments is $\simeq 0.52 \mathrm{pc}$. 


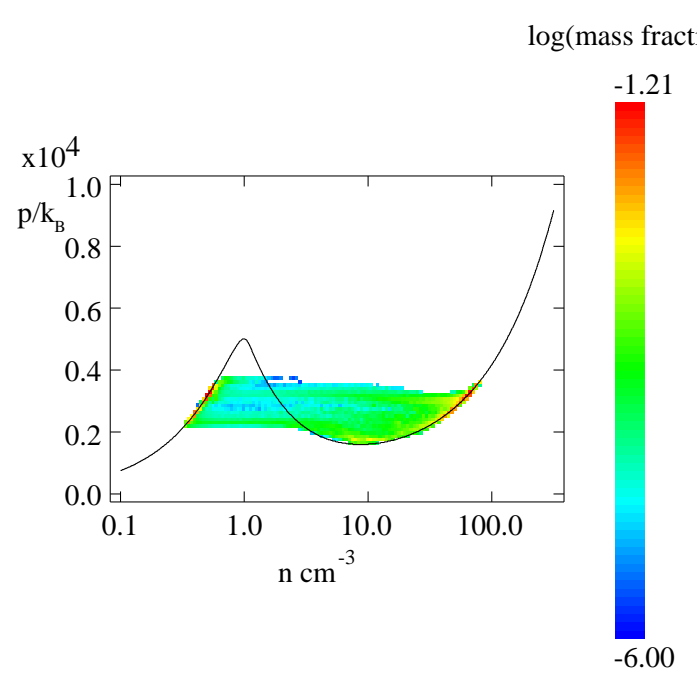

Figure 13. Distribution of mass fraction in the $n-p$ plane in the region $-10 \leq x \leq 15$ for the perturbed $2 \mathrm{D}$ MHD fast shock 5 at $t=25$ Myrs. The integrated mass fractions are: 0.3589 for warm gas, 0.5748 for cold gas and 0.066 for unstable gas.

more realistic initial field in shocks 8 to 11 , even for small values of the angle between the field and the shock normal.

\section{NUMERICAL CALCULATIONS}

In order to find out what happens to steady solutions that reach the unstable state, we carried out time dependent numerical calculations with the same AMR MHD code, MG, as in Wareing et al. (2016a). To keep things as simple as possible, these were two dimensional Cartesian calculations starting with a steady shock solution propagating in the $x$ direction. This is perturbed by imposing a periodic shift in the $x$ position of the shock and hence the whole solution. The initial solution is then given by

$\mathbf{p}(x, y)=\mathbf{p}_{s}\left[x+\sin \left(2 \pi y / y_{d}\right)\right]$,

where $\mathbf{p}_{s}(x)$ is the steady solution and $y_{d}$ is the width of the domain in the $y$ direction. The resulting initial states are shown in Figs. 10, 11 and 12. The upstream state was imposed at the right $x$ boundary and on the left the $x$ velocity was fixed at that of the end state with zero gradient for the other variables. The $y$ boundaries were periodic.

Fig. 10b shows the density for the hydrodynamic shock 1 at 20 Myrs. The instability has generated corrugations in the boundary between the warm and cold gas and variations in the cold gas density, which are then advected towards the left boundary. However, the density of the cold gas only varies from $100 \mathrm{~cm}^{-3}$ to $300 \mathrm{~cm}^{-3}$ and these regions are not in pressure equilibrium as can be seen from Fig. 10c. There is also warm gas next to cold gas in the region $y<4$ and $x<2$, but again these are not in pressure equilibrium and the warm phase is in the unstable region above the equilibrium curve. It is clear that the density variations in the cold gas will reduce as the pressure equilibrates and the unstable warm gas must turn into stable cold gas since the gas pressure is too high for a stable warm phase to exist. This is just telling us that such a shock cannot generate a (a)

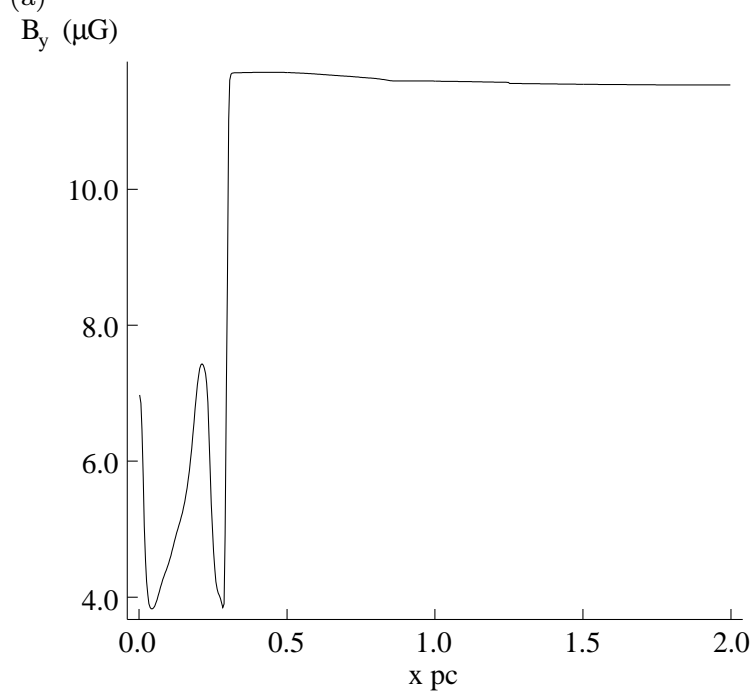

(b)

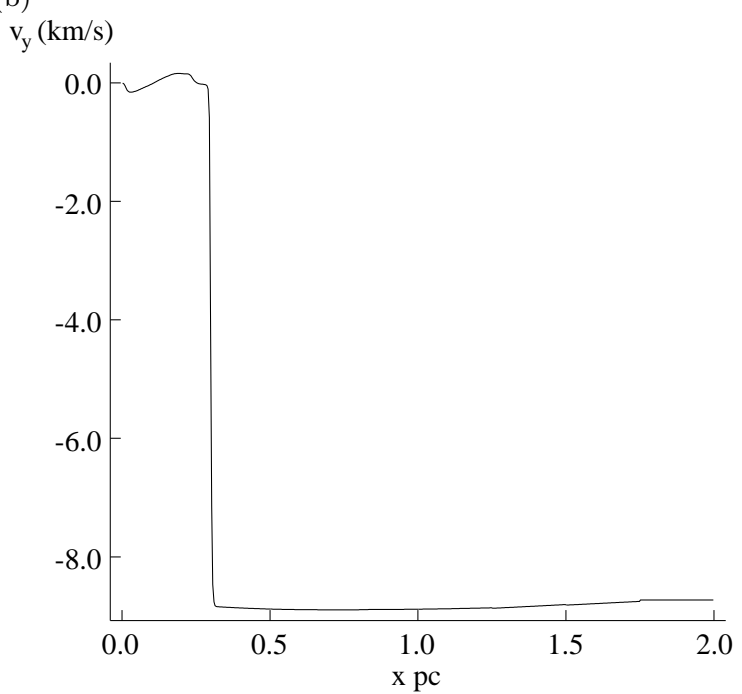

Figure 14. Region near the interface for Inoue \& Inutsuka (2009) case 1a (shock 11 in Table 1 ) at $t=60$ Myr. a) transverse magnetic field, b) transverse velocity. At this time the fast shock is at $x=$ 88.075 .

two-phase medium in pressure equilibrium, which is exactly what we would expect.

The difference between shocks 2 and 5 shown in Figs. 11 and 12 , is that shock 5 has a larger pre-shock magnetic field. As a result, the magnetic pressure dominates for $x<60$, whereas in shock 2 this does not happen until $x<15$. The disturbances caused by the instability are therefore able to distort the field in shock 2 to produce the ripples in the field lines in Fig. 11b, but not in shock 5.

In both cases the region near the left boundary consists of cold gas in pressure equilibrium with the warm gas, as can be seen from the fact that the filaments are invisible in the plots of the gas pressure in Figs. 11c and 12c. It is also in thermal equilibrium and is therefore a genuine two-phase medium. In both cases the mass fraction of the 
$\log (\mathrm{n})$
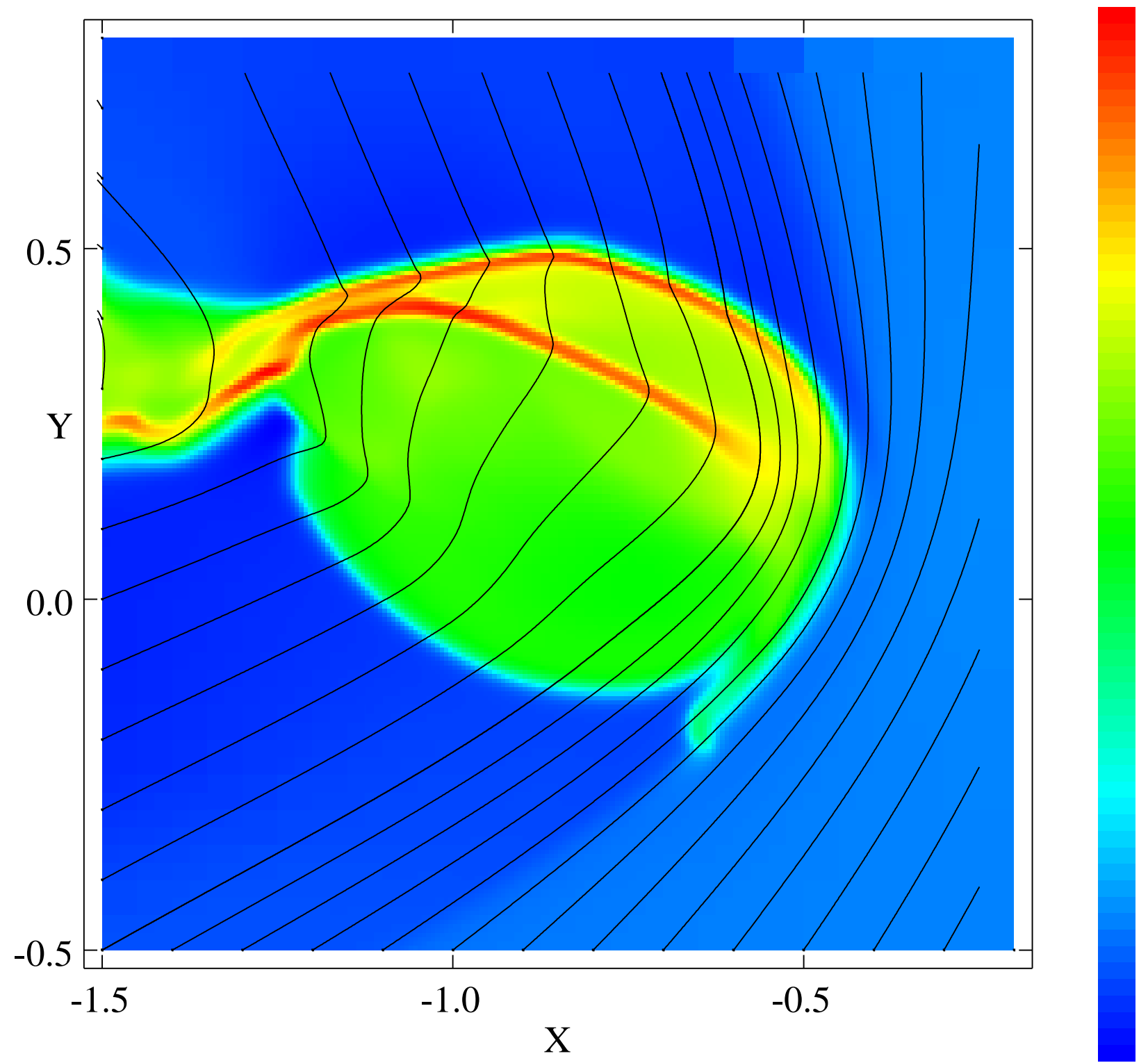

Figure 15. Log density and magnetic field lines for the shock-cloud interaction at $t=14.5$ Myrs. Distance is in units of the initial cloud radius $(200 \mathrm{pc})$.

unstable gas near the left boundary is less than $10 \%$ i.e. the gas has largely separated into stable warm and cold phases. This separation into warm and cold phase for shock 5 can clearly be seen in the plot of mass fraction in the $n-p$ plane shown in Fig. 13. Note that the amount of gas in this final state does not increase systematically since there is ouflow from the left boundary. In reality, the mass in the final state would increase with time irrespective of whether the shock is externally driven or due to a collision between two streams.
The thickness of the region in the two-phase state should increase by $0.434 \mathrm{pc} \mathrm{Myr}^{-1}$ for shock 2 and $2.29 \mathrm{pc} \mathrm{Myr}^{-1}$ for shock 5 .

The steady shock solutions are not a bad guide to what happens: the total pressure is close to the ram pressure, as we would expect from global momentum balance; the mean density is 3.83 compared to a steady value of 4.37 for shock 2 and 1.32 compared to 1.24 for shock 5. However, the gas pressures in the numerical calculations are $\simeq 3200$ in both 
cases, whereas we would expect 1818 for shock 2 and 4332 for shock 5 . As a consequence, the warm density and cold densities are $\simeq 0.5$ and $\simeq 80$ in both cases instead of 0.27 and 19 for shock 2 and 1.24 and 106 for shock 5 as required by the gas pressures in the steady solutions. These differences in the gas pressure are not surprising since the final state in the unsteady case is a two phase medium as opposed to an unstable single phase medium. Even though the mean density is roughly the same, the gas pressure in the two phase state depends on the fractions of warm and cold gas, which in turn depend on the time history of the instability.

The two phase medium with high density filamentary structures in Figs. 11 and 12 is similar to that generated by randomly perturbing an initially unstable state (Wareing et al. 2016a). Despite being produced in very different ways, both the separation, $5-10 \mathrm{pc}$, and width, $\simeq 0.5 \mathrm{pc}$, of the filaments are very similar. In neither case does the separation seem related to the initial perturbation, but it is a factor of a few times smaller than the thermal wavelength at the low density end of the unstable region. We might expect this wavelength to be favoured since Fig. 5a shows that this is the largest wavelength for which the growth rate is close to its maximum. We have not included thermal conduction, so there is no physical maximally unstable wavelength, although very short wavelengths are suppressed by numerical thermal conductivity.

Without self-gravity, this two-phase medium would not evolve as long as the total pressure remains constant. Selfgravity is not important on the scale of the filaments: the Jeans length in the filaments is $\simeq 12 \mathrm{pc}$ in both cases, which is much larger than their widths. However, there is the possibility of large scale gravitational collapse along the field as in Wareing et al. (2016a). The relevant timescale for this is the free-fall time for one dimensional collapse, $1 / \sqrt{\pi G \rho}$, which gives 25 Myr for shock 2 and 42.6 Myr for shock 5 . Wareing et al. (2016a) showed that most of the mass collects in a corrugated sheet perpendicular to the magnetic field, which can then collapse perpendicular to the field if the mass to flux ratio is large enough.

Fogerty et al. (2016) point out that the appropriate critical mass to flux ratio is the one for a field perpendicular to a plane layer in hydrostatic equilibrium

$\frac{\Sigma}{B}=\frac{1}{2 \pi \sqrt{G}}=616.25$ c.g.s.

where $\Sigma$ is the surface density of the layer (Nakano \& Nakamura 1978). The two-phase region will be supercritical if its width along the field is greater than

$W_{c}=100\left(\frac{B / 10^{6}}{n}\right)$ pc.

This gives $26 \mathrm{pc}$ for shock 1 and $76 \mathrm{pc}$ for shock 5 , so we clearly need long-lived, large scale flows for gravitational collapse.

This all assumes that the two-phase region is constrained from expanding perpendicular to the inflow. This is true for those simulations that impose periodic conditions at the boundaries without inflow: Koyama \& Inutsuka 2002; Heitsch et al. 2009; Inoue \& Inutsuka 2008, 2009. Audit \& Hennebelle (2005) used free boundary conditions for their purely hydrodynamic calculations, as did Fogerty et al.
(2016) who considered a parallel field with $\beta=10$. Since the initial shock was effectively hydrodynamic in both cases, it produced high enough densities for self-gravity to be significant despite the lack of constraint on the sideways expansion.

\subsection{Slow shocks}

So far we have only considered fast MHD shocks, but if they are due to a collision between two streams, then there must also be slow shocks. For example, in a plane symmetric collision between two streams the two fast shocks generate velocities perpendicular to the shock normal that have opposite signs. At the interface these velocities must be equal, which can only be accomplished by a slow shock or a fast rarefaction. In a plane collision the only possibility is a slow shock.

Inoue \& Inutsuka (2009) find clear evidence of slow shocks in some of their cases and not in others. In fact slow shocks must be present in all cases, but in some of them the shocks move so slowly that they are hard to resolve. For example, Fig. 14 shows the region near the interface for a one dimensional version of their case 1a: a collision with initial density 0.67 , velocity $20 \mathrm{~km} \mathrm{~s}^{-1}$, field $3 \mu \mathrm{G}$ at an angle of $15^{\circ}$ to the flow. Shock 11 in Table 1 is the steady fast shock generated by this collision if we ignore the slow shock. We can see that there is a slow shock at $x=0.3$ in which the transverse field and velocity decrease so that the transverse velocity vanishes at the interface. Note that the oscillations are due to the instabilities in the state upstream of the slow shock. Although the density behind the shock is $\simeq 1.7510^{3}$, the amount of mass involved is negligible. Since this is generally true, these shocks are of little significance, which is just as well since they are very hard to resolve in many cases: this calculation required 7 levels of AMR with a finest resolution of $410^{-3} \mathrm{pc}$.

\subsection{Shock-cloud interactions}

Van Loo et al. (2010) considered a plane fast shock interacting with a spherical warm cloud with density 0.45 in pressure equilibrium with a hot medium with density $n=0.01$. The shock sonic Mach number was 2.5 ( $\equiv 142.5 \mathrm{~km} \mathrm{~s}^{-1}$ ), the cloud radius was $200 \mathrm{pc}$ and the initial magnetic field was uniform with $\beta=1$. They used the heating and cooling prescription described Sánchez et al. (2002) which differs somewhat from the one in Koyama \& Inutsuka (2002) that we have considered here. For example, it is unstable for $n \geq 0.5$, rather $n=1$. However, we do not expect this to lead to a qualitative difference in the results.

They found that a slow shock formed at the boundary between the cloud and the ambient medium, but it only involved a significant amount of mass when the incident shock normal was parallel to the field: its effect was neglible even for an angle as small as $15^{\circ}$. Slow shocks can therefore only generate a significant amount of gas at high densities when the field and the shock normal are very closely aligned. In the general case with plausible values of the initial $\beta$, slow shocks will be unimportant and most of the material will end up in the two-phase state.

Fig. 15 shows the density and field lines in a very similar 
calculation to these: the density of external medium is $n=$ 0.01 , the thermal Mach number of the shock is 2.5 ( $\equiv 150.4$ $\mathrm{km} \mathrm{s}^{-1}$ ), the cloud radius, $R_{C}$, is $200 \mathrm{pc}$, the initial pressure is $3150.25 k_{B}$, the initial $\beta=1(B=3.3 \mu \mathrm{G})$, the field is parallel to the $x-y$ plane at an angle of $45^{\circ}$ to $x$ axis. The shock travels in the $-x$ direction. The domain is $-3 R_{c} \leq x, y, z \leq$ $3 R_{c}$ and 6 grid levels were used with a finest resolution of $1.25 \mathrm{pc}$, which is slightly better than the $1.67 \mathrm{pc}$ in Van Loo et al. (2010). The most significant difference is that the energy source function is given by equations (65) and (66).

The regions with density $\simeq 100$ are curved sheets about 5 pc thick and an extent of about 200 pc perpendicular to the $x-y$ plane. There is a region between these sheets that has $\beta<0.1$ and is in the unstable density range, but above the equilibrium curve, which we expect to cool and evolve into a two-phase medium. The sheets have slightly higher gas pressure than this unstable region and are accumulating mass. They are on a much larger scale than that of thermal instability in Figs. 11 and 12, which suggests that they are a result of the large-scale shock propagation rather than thermal instability.

The Jeans length defined by

$L_{J}=\left(\frac{\pi a^{2}}{G \rho}\right)^{1 / 2}$,

is $\simeq 10-20 \mathrm{pc}$ in the sheets, so that self-gravity is becoming significant. Gravity is likely to bring the sheets and the material between them together, which would make them close to supercritical according to equation (71), especially since the field is mostly not perpendicular to the sheets. They should then be subject to the gravitational instabilities considered by Van Loo et al. (2014).

One might have hoped that slow shocks would produce high densities, but there is no evidence that they play a significant role. This is consistent with the results in Van Loo et al. (2010) for this angle between the field and the shock normal.

Although this calculation and those in Van Loo et al. (2010) are interesting, there are two reasons why they must be regarded as indicative rather accurate solutions to the problem as posed. The first is that the resolution is not sufficient to resolve the scales on which the thermal instability appears in Figs. 11 and 12. The second is that the flow behind the incident shock is sub-fast, which means that the reflected shock propagates to upstream infinity. In our calculation it reaches the upstream boundary at 7.2 Myrs, after which the external flow is incorrect. This might not actually matter very much since the dynamic pressures in the external flow are too small to have much effect on the evolution of the cloud. We tested this by reducing the size of the domain after 12.25 Myrs and found that this made little difference to the flow in the cloud.

\section{SUMMARY AND CONCLUSIONS}

In this paper we have reworked the linear stability analysis in Field et al. (1969) using a combination of the HermiteBiehler theorem and Whitham's theory of wave hierarchies in Section 2, analysed its implications for the energy source in Koyama \& Inutsuka (2002) in Section 3 and described appropriate numerical calculations in Section 4.
Most of the results in Section 2 are already known, but our method simplifies the calculations considerably as well as establishing a simple relationship between the dispersion relation and the various physical processes. For example, we were able to write down the dispersion relation for MHD simply from a knowledge of the MHD wavespeeds and hence show that that the inclusion of a magnetic field has no effect on the stability. We also consider the stability of nonequilibrium states and show that the standard analysis is only quantitatively valid for shocks that are barely capable of triggering a transition to the cold phase.

Section 3 discusses the stability properties of both equilibrium and non-equilibrium states for the widely used energy source function suggested by Koyama \& Inutsuka (2002). We also computed a number of steady shock solutions, both with and without a magnetic field. These results confirm that for most plausible parameters, the final state lies on the unstable part of the equilibrium curve. This means that such shocks cannot exist, but it is clear that the end result must be a two-phase medium consisting of warm and cold phases with the gas pressures in equilibrium. This is confirmed by the numerical calculations of perturbed steady shock solutions in Section 4. The main point here is that the steady shock solutions are useful for analysing numerical calculations, even though they cannot exist in reality.

We considered a shock-cloud interacton similar to those in Van Loo et al. (2010). This large scale shock interaction produces dense sheets whose scale is determined by the size of the cloud rather than that of the thermal instability. They are sufficiently dense to collapse under their own gravity.

Finally, we have shown that slow shocks are unlikely to play a significant role in these kinds of flow. This is a pity since they are the only way of producing high densities in the presence of plausible magnetic fields.

\section{ACKNOWLEDGEMENTS}

We are grateful to an anonymous referee for helpful comments on the original version. This work was supported by the Science and Technology Facilities Council (STFC, Research Grant ST/P00041X/1). The calculations for this paper were performed on the DiRAC 1 Facility at Leeds jointly funded by STFC, the Large Facilities Capital Fund of BIS and the University of Leeds and on other HPC facilities at the University of Leeds. These facilities are hosted and enabled through the ARC HPC resources and support team at the University of Leeds, to whom we extend our grateful thanks. The DiRAC Data Centric system at Durham University was also used, operated by the Institute for Computational Cosmology on behalf of the STFC DiRAC HPC Facility (www.dirac.ac.uk). This equipment was funded by a BIS National E-infrastructure capital grant ST/K00042X/1, STFC capital grant ST/K00087X/1, DiRAC Operations grant ST/K003267/1, and Durham University. DiRAC is part of the National E-Infrastructure.

\section{REFERENCES}

Audit E., Hennebelle P., 2005, A\&A, 433, 1

Balbus S. A., 1986, ApJ, 303, L79

Begelman M. C., McKee C. F., 1990, ApJ, 358, 375 
Bhattacharyya S. P., Chapellat H., Keel L. H., 1995, p. 41, Chapter 1, Robust Control, Prentice-Hall PTR, Upper Saddle River, N.J.

Dudorov A.E., Stepanov C.E., Fomin S. O., Khaibrakhmanov, S.A., 2019, MNRAS, 487, 942

Falle S. A. E. G., Williams R. J. R., 2019, SIAM J. Appl. Math., 79,459

Field G. B., 1965, ApJ, 142, 531

Field G. B., Goldsmith D. W., Habing H. J., 1969, ApJ, 155, L149

Fogerty E., Frank A., Heitsch F., Carroll-Nellenback J., Haig C., Adams M., 2016, MNRAS, 460, 2016

Gnat O., Ferland G. J., 2012, ApJS, 199, 20

Heitsch F., Hartmann L. W., Slyz A. D., Devriendt J. E. G., Burket A., 2008a, ApJ, 674, 316

Heitsch F., Hartmann L. W., Burket A., 2008b, ApJ, 683, 786

Heitsch F., Stone J. M, Hartmann L. W., 2009, ApJ, 695, 248

Hennebelle P., Pérault M., 2000, A\&A, 359, 1124

Inoue T., Inutsuka S.-I., 2008, ApJ, 687, 303

Inoue T., Inutsuka S.-I., 2009, ApJ, 704, 161

Kim C.-G., Kim W.-T, Ostriker E. C.,2008, ApJ, 681, 1148

Koyama H., Inutsuka S.-I., 2000, ApJ, 532, 980

Koyama H., Inutsuka S.-I., 2002, ApJ, 564, L97

Koyama H., Inutsuka S.-I., 2004, ApJ, 602, L25

Liubarskii G. Ia., 1961, PMM, 25, 1041

McKee C. F., Ostriker J. P., 1977, ApJ, 218, 148

Nakano T., Nakamura T., 1978, PASJ, 30, 671

Parker E. N., 1953, ApJ, 117, 431

Sánchez-Salcedo F. J., Vásquez-Semadini E., Gazol, A, 2002, ApJ, 577,768

Schwarz J., McCray R., Stein, R. F., 1972, ApJ, 175, 673

Stern J., Hennawi J. F., Prochaska J. X., Werk J. K., 2016, ApJ, 830, 87

Tytarenko P. V., Williams R. J. R., Falle S. A. E. G., 2002, MNRAS, 337, 117

Van Loo S., Falle S. A. E. G., Hartquist T. W., 2010, MNRAS, 406, 1260

Van Loo S., Keto E., Zhang Q., 2014, ApJ, 789, 37

Vásquez-Semadini E., Gómez G. C., Jappsen A. K., BallesterosParedes J., Gonzáles R. F., Klessen R. S., 2007, ApJ, 657, 870

Wareing C. J., Pittard J. M., Falle S. A. E. G., Van Loo S., 2016a, MNRAS, 459, 1803

Wareing C. J., Pittard J. M., Falle S. A. E. G., 2016b, MNRAS, 465,2757

Wareing C. J., Pittard J. M., Falle S. A. E. G., 2017, MNRAS, 470,2283

Wareing C. J., Pittard J. M., Wright N. J., Falle S. A. E. G., 2018, MNRAS, 475, 3598

Wareing C. J., Pittard J. M., Falle S. A. E. G., 2019, MNRAS, 485,4686

Waters T., Proga D., 2019, ApJ, 875, 158

Whitham G. B., 1974, Linear and Non-linear Waves, New York, Wiley

Xia C., Keppens R., 2016, ApJ, 823, 22 